\title{
Dissipation, MHD and Radiation Effects on an Unsteady Convective Heat and Mass Transfer in a Darcy-Forcheimer Porous Medium
}

\author{
Moses S. Dada (Corresponding author) \\ Department of Mathematics, University of Ilorin, Ilorin, Nigeria \\ E-mail: msadada@unilorin.edu.ng
}

Funmilayo H. Adefolaju

Department of Mathematics, Afe Babalola University, Ekiti State, Nigeria

E-mail: adefolajufunmilayo@yahoo.com

Received: February 1, 2012 Accepted: February 17, 2012 Published: April 1, 2012

doi:10.5539/jmr.v4n2p110 URL: http://dx.doi.org/10.5539/jmr.v4n2p110

\begin{abstract}
This study investigates the effects of dissipation, magneto-hydrodynamics and thermal radiation on the natural convective heat and mass transfer of a viscous incompressible, gray absorbing-emitting fluid flowing past an impulsively started moving vertical plate in the Darcy-Forchheimer porous medium. The governing equations for the model are formulated in $\left(x^{*}, y^{*}, t^{*}\right)$ co-ordinates system with appropriate boundary conditions and the radiative heat flux takes the Rosseland diffusion approximation. The equations are simplified, non-dimensionalized and then solved using the Crank-Nicolsons method. The effects of Magnetic field, dissipation function, radiation-conduction and Forchheimer parameters with thermal Grashof, species Grashof, Prandtl, Schimdt and Darcy numbers are reported on the dimensionless velocity, temperature and species function distributions. Also, the variations of local skin friction, Nusselt and Sherwood numbers are computed.
\end{abstract}

Keywords: Viscous dissipation, MHD, Thermal radiation, Darcy-Forchheimer porous medium, Finite difference

\section{Introduction}

Thermal radiation heat transfer in combination with conduction convection and mass transfer play significant roles in the industrial designs involving high temperature processes such as in the industrial furnace systems, turbines, astrophysical flows, nuclear power plants, forest fire dynamics and fire spread in buildings (Anwar Beg, Zueco, Takhar \& Beg, 2008; Mansour, 1990; Modest, 1993). Soundalgekar \& Takhar (1993) considered the radiation free convective flow of an optically thin gray gas past a semi-infinite vertical plate. Hossain, Kutibuddin \& Tarkar (1999) studied the radiation interaction on combined forced and free convection across a horizontal cylinder and Chamkha, Tarkar \& Soundalgekar (2001) studied radiation effects on the free convection flow past a semi-finite vertical plate with mass transfer. Prasad, Reddy \& Muthueumaraswamy (2007) reported on the radiation and mass transfer effects on two-dimensional flow past an impulsively started infinite vertical plate. They observed that when the radiation parameter increases, the velocity and temperature decrease in the boundary layer.

The interest of some researchers were drawn to the hydromechanics of porous media since it was shown that temperature distribution in the fabric layers are strongly affected by moisture content and thermal radiation flux. A Darcian model of porous media is generally accurate for situations for low velocity where Reynold number is approximately less than ten. Beyond this value, inertial effects become significant and must be incorporated in mathematical models. Both Darcian and Darcy-Forchheimer (inertial) models have been employed extensively in radiative convection flow in porous media (Anwar Beg et al., 2008). Moreover, radiation effects is significant in the dynamics of fluid among others in chemical, environmental, mechanical and solar power engineering. Thus, Chamkha (1997) studied the effects of solar radiation on free convection in an isotropic, uniform porous medium supported by a vertical flat plate using a computational method. Mohammadein, Mansour, El Gaied \& Gorla (1998) employed a regular two-parameter perturbation analysis in studying radiative flux effects on free convection in a non-Darcian porous medium. Four flow regimes i.e adjacent to the isothermal surface, flow with a uniform heat flux, plane plume flow and the flow generated from a horizontal line energy source on a vertical adiabatic surface were studied. In all cases, radiation significantly affects flow. Chamkha, Takhar \& Beg (2004) studied the influence of thermal radiation on steady natural convection in a non-newtonian fluid past a wedge embedded in a porous medium using keller Box numerical scheme and reported among other things that the temperature increases 
with an increase in the radiation parameter. Recently, Anwar Beg et al. (2008) studied the network numerical simulation of impulsively-started transient radiation convection heat and mass transfer in a non-Darcy porous medium neglecting the effects of dissipation and magnetic field.

In the investigations mentioned above, viscous dissipation effects on an impulsively-started transient radiation-convection heat and mass transfer in the Darcy-Forchheimer porous medium is assumed to be negligible. Effects of viscous dissipation are important in geophysical flow and also in certain industrial operations A number of authors have considered viscous heating effects on newtonian flows. Mahajan \& Gebhart (1989) reported on the influence of viscous heating dissipation effects in a natural convective flow and showed that the heat transfer rates are reduced by an increase in the dissipation parameter. Tarkar, Beg \& Kumari (1998) used the implicit difference scheme and the Cogley-Vincenti-Giles non-gray model to simulate the radiation-convection gas flow in a non-Darcy porous medium with viscous heating effects. Also, Sangapatnam, Nandanoor \& Vallampati (2009) considered the radiation and mass transfer effects on MHD free convection flow past impulsively-started isothermal vertical plate with dissipation.

Also, effects of magnetic field on viscous incompressible fluid of electrically conducting is of importance in many applications such as extrusion of plastics in the manufacture of Rayon and Nylon, purification of crude oil, textile industries and so on. Zueco (2006) used network simulation method (NMS) to study the free convective MHD flow of viscous dissipative fluid along vertical plate subject to constant heat flux. Aydin \& Kaya (2009) analysed the problems the effects of MHD mixed convection of a viscous dissipation fluid about a permeable vertical flat plate applying numerical method and Palani \& Srikanth (2009) solved the problem of MHD flow past a semi-finite vertical plate with mass transfer using an implicit finite difference scheme of Crank-Nicolson type.

However, Little attention has been paid to investigating the coupling influences of dissipation, MHD and radiation on an unsteady convective heat and mass transfer in a Darcy-Forcheimer porous medium. Hence, the objective of the present investigation.

\section{Problem Formulation}

We consider an unsteady two-dimensional laminar natural convection flow of a viscous incompressible, dissipative, electrically conducting and radiating fluid past an impulsively started semi-infinite vertical plate in the presence of transverse magnetic field. The fluid is assumed to be gray, absorbing-emitting but non-scattering. The $y^{*}$-axis is taken perpendicular to the plate at the leading edge while the $x^{*}$-axis is chosen along the plate in the vertical upward direction. The origin of $x^{*}$-axis is taken to be at the leading edge of the plate. The gravitational acceleration $\mathrm{g}$ is acting downward. At time $t^{*}=0$, it is assumed that the plate and the fluid are at the same ambient temperature $T_{\infty}^{*}$ and the species concentration $C_{\infty}^{*}$. When $t^{*}>0$, the temperature of the plate and the species concentration is maintained to be $T_{w}^{*}$ (greater than $T_{\infty}^{*}$ ) and $C_{w}^{*}$ (greater than $C_{\infty}^{*}$ ) respectively.

Effect of viscous dissipation is considered in the binary mixture and assumed to be very small compared with other chemical species, which are present. A uniformly transverse magnetic field is applied in the direction of flow. It is further assumed that the interaction of the induced magnetic with the flow is considered to be negligible compared to the interaction of the applied magnetic field with the flow. The fluid properties are assumed to be constant except for the body forces terms in the momentum equation which are approximated by Boussinesq relations. Thermal radiation is assumed to be present in the form of an uni-directional flux in the $y^{*}$ direction i.e $q_{r}$ is transverse to the vertical surface.

The Rosseland diffusion flux is used and defined following Modest (1993) as follows:

$$
q_{r}=-\frac{4 \sigma}{3 k^{1}} \frac{\partial T^{* 4}}{\partial y^{*}}
$$

where $\sigma$ is the Stefan-Boltzmann constant and $k^{1}$ is the mean absorption coefficient. It should be noted that by using the Rosseland approximation, the present analysis is limited to optically thick fluid.

Under the Boussinesq approximation, the boundary layer equations for mass, momentum, energy and species conservation along velocity components $u^{*}$ and $v^{*}$ in the $x^{*}$ and $y^{*}$ directions at time $t^{*}$ can be shown to take the form:

$$
\begin{gathered}
\frac{\partial u^{*}}{\partial x^{*}}+\frac{\partial v^{*}}{\partial y^{*}}=0 \\
\frac{\partial u^{*}}{\partial t^{*}}+u^{*} \frac{\partial u^{*}}{\partial x^{*}}+v^{*} \frac{\partial u^{*}}{\partial y^{*}}=v \frac{\partial^{2} u^{*}}{\partial y^{* 2}}+g \beta\left(T^{*}-T_{\infty}^{*}\right)+g \beta^{*}\left(C^{*}-C_{\infty}^{*}\right) \\
-\frac{\sigma}{\rho} B_{O}^{2} u^{*}-\frac{v}{k} u^{*}-\frac{b}{k} u^{* 2}
\end{gathered}
$$




$$
\begin{gathered}
\frac{\partial T^{*}}{\partial t^{*}}+u^{*} \frac{\partial T^{*}}{\partial x^{*}}+v^{*} \frac{\partial T^{*}}{\partial y^{*}}=\alpha \frac{\partial^{2} T^{*}}{\partial y^{* 2}}+\frac{v}{C_{p}}\left[\frac{\partial u^{*}}{\partial y^{*}}\right]^{2}-\frac{1}{\rho C_{p}} \frac{\partial q_{r}}{\partial y^{*}} \\
\frac{\partial C^{*}}{\partial t^{*}}+u^{*} \frac{\partial C^{*}}{\partial x^{*}}+v^{*} \frac{\partial C^{*}}{\partial y^{*}}=D\left[\frac{\partial^{2} C^{*}}{\partial y^{* 2}}\right]
\end{gathered}
$$

The corresponding initial and boundary conditions are prescribed as follows:

$$
\begin{aligned}
& t^{*} \leq 0, \quad u^{*}=0, \quad v^{*}=0, \quad T^{*}=T_{\infty}^{*}, \quad C^{*}=C_{\infty}^{*} . \\
& t^{*}>0, \quad u^{*}=u_{0}^{*}, \quad v^{*}=0, \quad T_{w}^{*}=T_{\infty}^{*}, \quad C_{w}^{*}=C_{\infty}^{*} \quad \text { at } y^{*}=0 \\
& u^{*}=0, \quad T^{*}=T_{\infty}^{*}, \quad C^{*}=C_{\infty}^{*} \quad \text { at } x^{*}=0 \\
& u^{*} \rightarrow 0, \quad T^{*} \rightarrow T_{\infty}^{*} \quad C^{*} \rightarrow C_{\infty}^{*} \quad \text { as } y^{*} \rightarrow \infty
\end{aligned}
$$

where $\sigma$ is the Stefan-Boltzmann constant, $\beta$ is mass transfer coefficient of expansion, $v$ is the kinematic viscosity of the grey fluid, $T^{*}$ is the temperature of the fluid in the boundary layer, $\mathrm{g}$ is the gravitational acceleration, $k^{1}$ is the mean absorption coefficient of thermal expansion, $C^{*}$ is the species concentration, $b$ is the forchheimer parameter of the medium, $\mathrm{k}$ is the permeability, $\alpha$ is the thermal diffusivity, $\mathrm{D}$ is the species diffusivity, ()$_{w}$ denotes condition at the wall, (vertical surface) and ()$_{\infty}$ denotes condition in the free stream (outside the boundary layer).

As express by Rapits \& Perdikis (2004), the quartic temperature function $T^{* 4}$ can be expanded in Taylor series and neglecting higher order terms if the temperature differences within the flow are sufficiently small as:

$$
T^{* 4} \approx 4 T_{\infty}^{* 3} T^{*}-3 T_{\infty}^{* 4}
$$

Substituting the above equation (7) into the energy equation (4) gives

$$
\frac{\partial T^{*}}{\partial t^{*}}+u^{*} \frac{\partial T^{*}}{\partial x^{*}}+v^{*} \frac{\partial T^{*}}{\partial y^{*}}=\alpha \frac{\partial^{2} T^{*}}{\partial y^{* 2}}+\frac{v}{C_{p}}\left[\frac{\partial u^{*}}{\partial y^{*}}\right]^{2}+\frac{16 \sigma T_{\infty}^{* 3}}{3 k^{1} \rho C_{p}} \frac{\partial^{2} T^{*}}{\partial y^{* 2}}
$$

On introducing the following non-dimensional quantities

$$
\begin{array}{r}
X=\frac{x^{*} u_{0}^{*}}{v}, Y=\frac{y^{*} u_{0}^{*}}{v}, U=\frac{u^{*}}{u_{0}^{*}}, V=\frac{v^{*}}{u_{0}^{*}}, E_{c}=\frac{u_{0}^{* 2}}{C_{p}\left(T_{w}^{*}-T_{\infty}^{*}\right)} \\
t=\frac{t^{*} u_{0}^{* 2}}{v}, T=\frac{T^{*}-T_{\infty}^{*}}{T_{w}^{*}-T_{\infty}^{*}}, C=\frac{C^{*}-C_{\infty}^{*}}{C_{w}^{*}-C_{\infty}^{*}}, R e=\frac{u_{0}^{*} L}{v} \\
P r=\frac{v}{\alpha}, S c=\frac{v}{D}, D a=\frac{k}{L^{2}}, F s=\frac{b}{L}, N=\frac{k^{1} k}{4 \sigma T_{\infty}^{* 3}} \\
M=\frac{\sigma B_{0}^{2} v}{\rho U_{0}^{* 2}}, G r=\frac{g \beta v\left(T_{w}^{*}-T_{\infty}^{*}\right)}{u_{0}^{* 3}}, G m=\frac{g \beta^{*} v\left(C_{w}^{*}-C_{\infty}^{*}\right)}{u_{0}^{* 3}}
\end{array}
$$

where Ec is the Eckert number, Re is the Reynold number, Pr is the Prandtl number, Sc is the Schimdt number, Da is the Darcy number, Fs is the Forchheimer inertia number, $\mathrm{N}$ is the conduction-Radiation heat transfer parameter, $\mathrm{M}$ is the Magnetic field parameter, Gr is the thermal Grashof number, Gm is the species Grashof number while $\mathrm{U}$ and $\mathrm{V}$ are dimensionless velocity components in $\mathrm{X}$ - and $\mathrm{Y}$ - dimensionless directions respectively, $\mathrm{t}$ is the dimensionless time, $\mathrm{T}$ is the dimensionless temperature function and $\mathrm{C}$ is the dimensionless concentration function,

Applying these non-dimensional quantities (9), the set of equations (2), (3), (5), and (8) reduces to the following nondimensional equations

$$
\begin{gathered}
\frac{\partial U}{\partial X}+\frac{\partial V}{\partial Y}=0 \\
\frac{\partial U}{\partial t}+U \frac{\partial U}{\partial X}+V \frac{\partial U}{\partial Y}=G r T+G m C+\frac{\partial^{2} U}{\partial Y^{2}}-M U-\frac{U}{R e^{2} D a}-\frac{F s U^{2}}{D a R e} \\
\frac{\partial T}{\partial t}+U \frac{\partial T}{\partial X}+V \frac{\partial T}{\partial Y}=\frac{1}{P r}\left[1+\frac{4}{3 N}\right] \frac{\partial^{2} T}{\partial Y^{2}}+E c\left[\frac{\partial U}{\partial Y}\right]^{2} \\
\frac{\partial C}{\partial t}+U \frac{\partial C}{\partial X}+V \frac{\partial C}{\partial Y}=\frac{1}{S c} \frac{\partial^{2} C}{\partial Y^{2}}
\end{gathered}
$$


The corresponding initial and boundary conditions take the form

$$
\begin{aligned}
& t \leq 0, \quad U=0, \quad V=0, \quad T=0, \quad C=0 . \\
& t>0, \quad U=1, \quad V=0, \quad T=1, \quad C=1 \quad \text { at } Y=0 \\
& U=0, \quad T=0, \quad C=0 \quad \text { at } X=0 \\
& U \rightarrow 0, \quad T \rightarrow 0 \quad C \rightarrow 0 \quad \text { as } Y \rightarrow \infty
\end{aligned}
$$

For the purpose of energy systems engineering thermal designs, attention is drawn to skin friction, Nusselt number as well as Sherwood number in order to provide a good estimate of the basic heat and mass transfer process in the regime of the flow. As reported by Modest [16], the local values of skin friction, Sherwood number and Nusselt number are respectively computed as follows:

$$
\begin{aligned}
\tau_{x} & =-\left[\frac{\partial U}{\partial Y}\right]_{Y=0} \\
S h_{x} & =-X\left[\frac{\partial C}{\partial Y}\right]_{Y=0} \\
N u_{x} & =-X\left[\frac{\partial T}{\partial Y}\right]_{Y=0}
\end{aligned}
$$

and their averaging values are defined respectively as

$$
\begin{aligned}
& \bar{\tau}_{x}=-\int_{0}^{1}\left[\frac{\partial U}{\partial Y}\right]_{Y=0} d X \\
& \overline{S h}_{x}=-\int_{0}^{1}\left[\frac{\partial C}{\partial Y}\right]_{Y=0} d X \\
& \overline{N u}_{x}=-\int_{0}^{1}\left[\frac{\partial T}{\partial Y}\right]_{Y=0} d X
\end{aligned}
$$

\section{Numerical Technique}

In order to solve these unsteady coupled non-linear partial differential equations (10) - (13) with the boundary conditions (14), we employed an implicit finite difference scheme of the Crank-Nicolson type. This method has been extensively developed in recent years and remains one of the best reliable methods for solving partial differential equation. The partial differential equations are converted to difference equation. The Crank-Nicolson method converges fast and is unconditionally stable. It has been used in several heat transfer, radiation and convection flow problems. Prasad et al. (2007) used Crank-Nicolson scheme to analyse the transient convective heat and mass transfer with thermal radiation effects along a vertical impulsively started plane. Among other authors that used this method is Sangapatnam et al. (2009) who investigated the thermal radiation and mass transfer effects on MHD free convection dissipative fluid flow past impulsively-started vertical plate.

The co-ordinate $(X, Y, t)$ of the mesh points of the solution domain is defined by $X=i \Delta X, Y=j \Delta Y$ and $t=k \Delta t$ where $i, j, k$ are positive integers and The values of $U$ at these mesh points denoted by $U(i \Delta X, j \Delta Y, k \Delta t)=U_{i, j}^{k}$. The finite difference equations corresponding to these equations are given as follows:

$$
\begin{gathered}
\frac{\left[U_{i, j}^{k+1}-U_{i-1, j}^{K+1}+U_{i, j}^{k}-U_{i-1, j}^{k}+U_{i, j-1}^{k+1}-U_{i-1, j-1}^{k+1}+U_{i, j-1}^{k}-U_{i-1, j-1}^{k}\right]}{4 \Delta X}+ \\
\frac{\left[V_{i, j}^{k+1}-V_{i, j-1}^{k+1}+V_{i, j}^{k}-V_{i, j-1}^{k}\right]}{2 \Delta Y}=0 \\
\frac{\left[U_{i, j}^{k+1}-U_{i, j}^{k}\right]}{\Delta t}+U_{i, j}^{k} \frac{\left[U_{i, j}^{k+1}-U_{i-1, j}^{k+1}+U_{i, j}^{k}-U_{i-1, j}^{k}\right]}{2 \Delta X}+V_{i, j}^{k} \frac{\left[U_{i, j+1}^{k+1}-U_{i, j-1}^{k+1}+U_{i, j+1}^{k}-U_{i, j-1}^{k}\right]}{4 \Delta Y}= \\
G r \frac{\left[T_{i, j}^{k+1}+T_{i, j}^{k}\right]}{2}+G m \frac{\left[C_{i, j}^{k+1}+C_{i, j}^{k}\right]}{2}+\frac{\left[U_{i, j-1}^{k+1}-2 U_{i, j}^{k+1}+U_{i, j+1}^{k+1}+U_{i, j-1}^{k}-2 U_{i, j}^{k}+U_{i, j+1}^{k}\right]}{2(\Delta Y)^{2}}- \\
\frac{1}{D a R e^{2}} \frac{\left[U_{i, j}^{k+1}+U_{i, j}^{k}\right]}{2}-\frac{F s}{D a R e} U_{i, j}^{k} \frac{\left[U_{i, j}^{k+1}+U_{i, j}^{k}\right]}{2}-M \frac{\left[U_{i, j}^{k+1}+U_{i, j}^{k}\right]}{2}
\end{gathered}
$$




$$
\begin{gathered}
\frac{\left[T_{i, j}^{k+1}-T_{i, j}^{k}\right]}{\Delta t}+U_{i, j}^{k} \frac{\left[T_{i, j}^{k+1}-T_{i-1, j}^{k+1}+T_{i, j}^{k}-T_{i-1, j}^{k}\right]}{2 \Delta X}+V_{i, j}^{k} \frac{\left[T_{i, j+1}^{k+1}-T_{i, j-1}^{k+1}+T_{i, j+1}^{k}-T_{i, j-1}^{k}\right]}{4 \Delta Y}= \\
\frac{1}{P r}\left[1+\frac{4}{3 N}\right] \frac{\left[T_{i, j-1}^{k+1}-2 T_{i, j}^{k+1}+T_{i, j+1}^{k+1}+T_{i, j-1}^{k}-2 T_{i, j}^{k}+T_{i, j+1}^{k}\right]}{2(\Delta Y)^{2}}+E C\left[\frac{\left[U_{i, j+1}^{k}-U_{i, j}^{k}\right.}{2 \Delta Y}\right]^{2} \\
\frac{\left[C_{i, j}^{k+1}-C_{i, j}^{k}\right]}{\Delta t}+U_{i, j}^{k} \frac{\left[C_{i, j}^{k+1}-C_{i-1, j}^{k+1}+C_{i, j}^{k}-C_{i-1, j}^{k}\right]}{2 \Delta X}+V_{i, j}^{k} \frac{\left[C_{i, j+1}^{k+1}-C_{i, j-1}^{k+1}+C_{i, j+1}^{k}-C_{i, j-1}^{k}\right]}{4 \Delta Y}= \\
\frac{1}{S c} \frac{\left[C_{i, j-1}^{k+1}-2 C_{i, j}^{k+1}+C_{i, j+1}^{k+1}+C_{i, j-1}^{k}-2 C_{i, j}^{k}+C_{i, j+1}^{k}\right]}{2(\Delta Y)^{2}}
\end{gathered}
$$

The region of integration considered is a rectangle with sides $X_{\max }=1$ and $Y_{\max }=5.25$ where $Y_{\max }$ corresponds to $Y=\infty$ which is outside the momentum, thermal and concentration boundary layers. The subscript $i$ implies the grid point along the $X$-direction, $j$ along the $Y$-direction and $k$ along the $t$-direction. We therefore divide $\mathrm{X}$ and $\mathrm{Y}$ into $\mathrm{M}$ and $\mathrm{N}$ grid spacing respectively. The mesh sizes are $\Delta X=0.1, \Delta Y=0.25$ and $\Delta t=0.01$.

In the computations, as discussed in Sangapatnam et al. (2009) and Palani et al. (2009), the coefficients $U_{i, j}^{k}$ and $V_{i, j}^{k}$ appearing in the difference equation are treated as constants in any one time step. The values of $\mathrm{C}, \mathrm{T}, \mathrm{U}$ and $\mathrm{V}$ are known at all grid point at $t=0$ from the initial conditions. The valus of $\mathrm{C}, \mathrm{T}, \mathrm{U}$ and $\mathrm{V}$ at time level $(k+1)$ are calculated using the known values at previous time level $(k)$.

Hence, the finite difference equation forms a tridiagonal system of equations at every internal nodal point on a particular $i-$ level which is solved with the aid of Matlab package using Thomas algorithm as discussed in Carnahan, Luther \& Willkes (1969).

Thus, we calculate the values of $\mathrm{C}$ and $\mathrm{T}$ at every nodal point for a particular $i$ at $(k+1)$ th time level and the results were used in $\mathrm{U}$ at $(k+1) t h$ time level. The values of $\mathrm{V}$ are also calculated at every nodal point explicitly on a particular $i-$ level at $(k+1)$ th time level. In this way, the values of $\mathrm{C}, \mathrm{T}, \mathrm{U}$, and $\mathrm{V}$ are known at all grid point at time level $(k+1)$ in the region. The process is repeated several times for various $i$-level until the required time is reached. To test the accuracy of the results, we have compared the velocity profile against Y for various thermal Grashof number, Species Grashof number, Schmidt number and Conduction-radiation parameter with the Crank-Nicolson computation of Prasad et al. (2007) by setting the values $M=0, D a \rightarrow \infty\left(D a=10^{9}\right)$ and $F s=0$ in our finite difference equations and the mesh sizes are taken to be $\Delta X=0.1, \Delta Y=0.25$ and $\Delta t=0.01$. The velocity profiles obtained in Figure 1 for $X=1$ are in agreement with available solution of Prasad et al. (2007).

\section{Discussion of Results}

Series of computation has been carried out for the effects of controlling thermophysical and hydrodynamic parameters on the velocity, temperature, concentration as well as local skin friction, Nusselt number and sherwood number. The present analysis concerns the case of optically thick boundary layers Default values for parameters are: $\mathrm{Pr}=0.71$ (air), $S c=0.6, N=3.0, M=1.0, E c=0.001, G r=20, G m=20, D a=0.1, F s=0.1, R e=1$. All the graphs correspond to the default values except otherwise stated on the graph.

Figure 2 shows the effects of Gr, Gm and M on the dimensionless velocity profile against Y co-ordinate. An increase in $\mathrm{Gr}$ or Gm causes a rise in the velocity profiles, a rise in Grashof number boost the buoyancy force. The increase shows a rapid rise in the velocity near the wall and descends towards zero. A rise in $M$ decreases the velocity profile which is in agreement with the fact that the presence of magnetic field reduces the force on the free convective flow.

Figures 3 and 4 illustrate the effects of the magnetic field (M) and the viscous dissipation function (Ec) on the dimensionless velocity profile. An increase in the magnetic field causes reduction in the velocity profile as evident in figure 3 . The presence of magnetic field on an electrically conducting fluid results in increasing the Lorentz force which opposes and retards the motion of a fluid. Similarly, in figure 4, we observed that increasing dissipation function (Ec) produces a rise in the velocity profile. The dissipation function accelerates energy in the fluid motion as a result increasing the buoyancy force. Different values of magnetic field on the temperature and species concentration profiles are presented in figures 5 and 6. Temperature increases slightly with the increasing value of magnetic field parameter (M) for both the temperature and species concentration.

Figures 7 and 8 show the effect of Conduction-radiation and Darcy number on the temperature profile. A rise in $\mathrm{N}$ causes a significant fall in the temperature values from the highest value at the wall $(Y=0)$ across the boundary layer to the free stream. Thus, greater value of $\mathrm{N}$ corresponds to smaller radiation flux and the minimum temperature are observed for $N=5.0$ in figure 7. Thermal radiation thereby reduces the rate of energy transport to the fluid. In figure 8, as Darcy number increases, a rise in temperature profile is experienced. This rise temperature profile means that conduction heat transfer is more prevalence than convection heat transfer. 
The effects of Schmidt number (Sc) on the velocity and temperature profiles are shown in figures 9 and 10 . It is noticed that the velocity and temperature reduce due to a rise in Sc. Figure 10 shows that temperature decelerates sharply with an increase in Sc, which implies a reduction in diffusivity of the chemical molecular. The concentration profile lower from its maximum concentration at $Y=0$ to zero in the free stream.

Figure 11 shows the influence of Sc on the concentration profile. With larger Sc, fluid experiences lower diffusion properties i.e, concentration boundary layer will be thinner than the velocity boundary layer thickness. A rise in Sc from 0.75 through 0.95 and 1.3 to 2.0 produces a decrease in concentration. The importance of this result may be applicable in regulating the temperatures in energy systems.

Figures 12, 13 and 14 show the influence of Gr, Gm and Pr on the dimensionless temperature profile with Y at time $t=1$. The influence of thermal Grashof number Gr on the temperature profile implies the relative effects of the thermal buoyancy force to the viscous hydrodyanamic force in the boundary layer regime. An increase in $\mathrm{Gr}$ (i.e for $\mathrm{Gr}=100$, 200, 300 and 400) gives a decrease in the temperature throughout the porous regime. The temperature distribution decay smoothly from 1 at the wall $(Y=0)$ to zero at the edge of the boundary layer. The influence of the species Grashof number is similar to that of $\mathrm{Gr}$ as shown in figure 13. Again temperatures are seen to fall with rise (i.e from 20 to 500 ) in $\mathrm{Gm}$. There is more gradual reduction in temperatures. The influence of Prandtl number Pr on the temperature profile is seen in figure 14. Pr is the ratio of momentum diffusivity to thermal diffusivity. The numerical results shows that, as Prandtl number increases, a decrease in the thermal boundary layer thickness is experienced and in general, lowing the average temperature. This is because smaller value of Pr increases the thermal conductivity of the fluid temperatures across the boundary layer. Higher values of Pr reaches zero faster.

Figure 15 shows the influence of Pr on the velocity profile. An increase in Pr reduces the velocity, then the velocity across the boundary layer reaches zero faster. The influence of conduction-radiation parameter $\mathrm{N}$ on the velocity profile is shown in figure 16. As $\mathrm{N}$ increases, considerable reduction is observed in the velocity profile from the peak value at the wall $(Y=0)$ across the boundary layer regime to the free stream $Y \rightarrow \infty$, at which the velocity are negligible for any value of $\mathrm{N}$. Reduction in velocity is accompanied by reduction in velocity layers.

Figure 17 illustrates the effects of Fs on the velocity profile. As Fs decreases, we experience great increase in the velocity profile. In the momentum equation, the quadratic drag term $-\left[\frac{F s}{D a R e}\right] U^{2}$ is directly proportional to Fs. This implies that, as Fs increases from 0.2 to 20 , we observe a great fall in velocity component. Figures 18 shows the effects of the thermal Grashof number $\mathrm{Gr}$ and the conduction-radiation parameter $\mathrm{N}$ on the concentration profile. As Gr increases from 0.4 to 75 in figure 18, we observed a great decrease in the species concentration in the boundary layer.

Figures 19, 20 and 21 illustrates the variation of the local skin friction, local Nusselt number and the local Sherwood number with streamwise distance $X$ for various values of the Eckert number. In figure 19, it is observed that local skin friction increases as Ec increases. In figure 20, the local Nusselt number for different values of Ec is presented. Nusselt number is the ratio of convective heat transfer coefficient to conductive heat transfer coefficient. It is noticed that Nusselt number decreases with greater viscous dissipative heat. That is heat transfer rates are reduced by an increase in dissipation parameter. Sherwood number is the ratio of convective mass transfer coefficient to diffusive mass transfer coefficient. It is noticed in figure 21 that the sherwood numbers increases due to the presence of higher viscous dissipative heat.

\section{Conclusion}

This study deals with the effects of dissipation, MHD and thermal radiation on the unsteady convective heat and mass transfer in the Darcy-Forchheimer porous medium. The equations governing the model were highlighted and nondimensionalised. The Rosseland diffusion flux has been used to simulate radiative flux.

The mathematical equations to the problem were then solved using Crank-Nicolson implicit finite difference method. Series of computation was carried out to study graphically the effects of the controlling thermophysical parameters which are magnetic field $(\mathrm{M})$, dissipation function $(\mathrm{Ec})$, conduction radiation parameter $(\mathrm{N})$, Forchheimer parameter (Fs), thermal grashof number (Gr), species Grashof number (Gm), Prandtl number (Pr), Schmidtl number (Sc), Darcy number (Da) on the dimensionless velocity, temperature and concentration profiles as well as the local skin friction, local Nusselt number and local Sherwood number. It is noticed that temperature and concentration increase slightly with increased Magnetic field parameter while the presence of magnetic field has a retarding effect on the velocity profile. A rise in the conductionradiation parameter causes reduction in the velocity profile while a rise in the dissipation function induces a considerable rise in velocity $(\mathrm{U})$. Also, increase in the Prandtl number, Schmidtl number, species Grashof number, thermal Grashof number and the conduction-radiation parameter causes the temperature to reduce, while a rise in the Darcy number Da causes a rise in temperature along and normal to the wall. Increase in Forchheimer number reduces the velocity profile. Shear stress was found to increase with a rise in Ec at the wall while the Local Nusselt number increased with reduction in Ec. Sherwood number increases with a rise in Ec. 


\section{References}

Anwar Beg, O., Zueco J., Takhar, H. S., \& Beg, T. A. (2008). Network numerical simulation of impulsively-started transient radiation-convection heat and mass transfer in a saturated non-Darcy porous medium, Nonlinear Analysis, Modeling and Control,13(3), 281-303.

Aydin O., \& Kaya A. (2009). MHD mixed convection of a viscous dissipating fluid about a permeable vertical flat plate. Applied Mathematical Modelling, 33(1), 4086-4096. http://dx.doi.org/10.1016/j.apm.2009.02.002

Carnahan B., Luther H. A., \& Willkes J. O. (1969). Applied Numerical Method. John Wiley and Sons,New York, USA.

Chamkha, A. J. (1997). Solar radiation assisted natural convection in a uniform porous medium supported by a vertical flat plate. ASME, Journal of Heat Transfer, 119, 89-96. http://dx.doi.org/10.1115/1.2824104

Chamkha, A. J., Takhar, H. S., \& Beg, A. (2004). Radiation free convective non-newtonian fluid flow past a wedge embedded in a porous medium. International Journal of Fluid Mechanics Reseach, 31, 101-115. http://dx.doi.org/10.1615/InterJFluidMechRes.v31.i2.10

Chamkha, A. J., Tarkar, H. S., \& Soundalgekar, V. M. (2001). Radiation effects on the free convection flow past a semi-infinite vertical plate with mass transfer. Chemical Engeneering Journal, 84 (3), $335-342$. http://dx.doi.org/10.1016/S1385-8947(00)00378-8

Hossain, M. A, Kutibuddin M., \& Tarkar H. S. (1999). Radiation interaction on combined forced and free convection across a horizontal cylinder, International Journal of Applied Mechanics and Engineering, 4, 219-235.

Mahajan, R. L., \& Gebhart, B. B. (1989). Viscous dissipation effects in buoyancy-induced flows. International Journal of Heat and Mass Transfer, 7, 1380-1382.

Mansour, M. H. (1990). Radiative and free convection effects on the oscillatory flow past a vertical plate. Journal of Astrophysics Space Science, 166(2), 169-275.

Modest, M. F. (1993). Radiation Heat Transfer. MacGraw-Hill, New York.

Mohammadein, A. A., Mansour, M. A., El Gaied, S. M., \& Gorla, R. S. R. (1998). Radiative effects on natural convection flows in a porous media. Transport Porous Media, 32(3), 263-283.

Palani G., \& Srikanth U. (2009). MHD flow past a semi-infinite vertical plate with mass transfer. Nonlinear Analysis Modelling and Control, 14(3), 345-356.

Prasad, R. V, Reddy, B. N., \& Muthueumaraswamy, R. (2007). Radiation and mass transfer effects on two-dimensional flow past an impulsively started infinite vertical plate. International Journal of Thermal Science, 46(12), 1251-1258. http://dx.doi.org/10.1016/j.ijthermalsci.2007.01.004

Rapits A., \& Perdikis C. (2004). Unsteady flow through a highly porous medium in the presence of radiation. Transport Porous Media, 57(2), 171-179. DOI: 10.1023/B:TIPM.0000038262.65594.e8

Sangapatnam, S., Nandanoor, R., \& Vallampati, R. P. (2009). Radiation and mass transfer effects on MHD free convection flow past impulsively-started isothermal vertical plate with dissipation. Thermal Science, 13(2), 171-181.

Soundalgekar V. M., \& Takhar H. S. (1993). Radiation free convective flow of an optically thin gray gas past a semiinfinite vertical plate. Modelling Measurement and Control, B51, 31-40.

Tarkar, H. S, Beg, O. A., \& Kumari, M. A. (1998). Computational analysis of coupled radiation-convection dissipative non-grey gas flow in a non-Darcy porous medium using the keller-Box implicit scheme. International Journal of Energy Research, 22,141-159.

Zueco J. (2006). Numerical study of an unsteady free convective magnetohydrodynamic flow of a dissipative fluid along a vertical plate subject to constant heat flux. International Journal of Engineering Science, 44, 1380-1393. http://dx.doi.org/10.1016/j.ijengsci.2006.08.006 


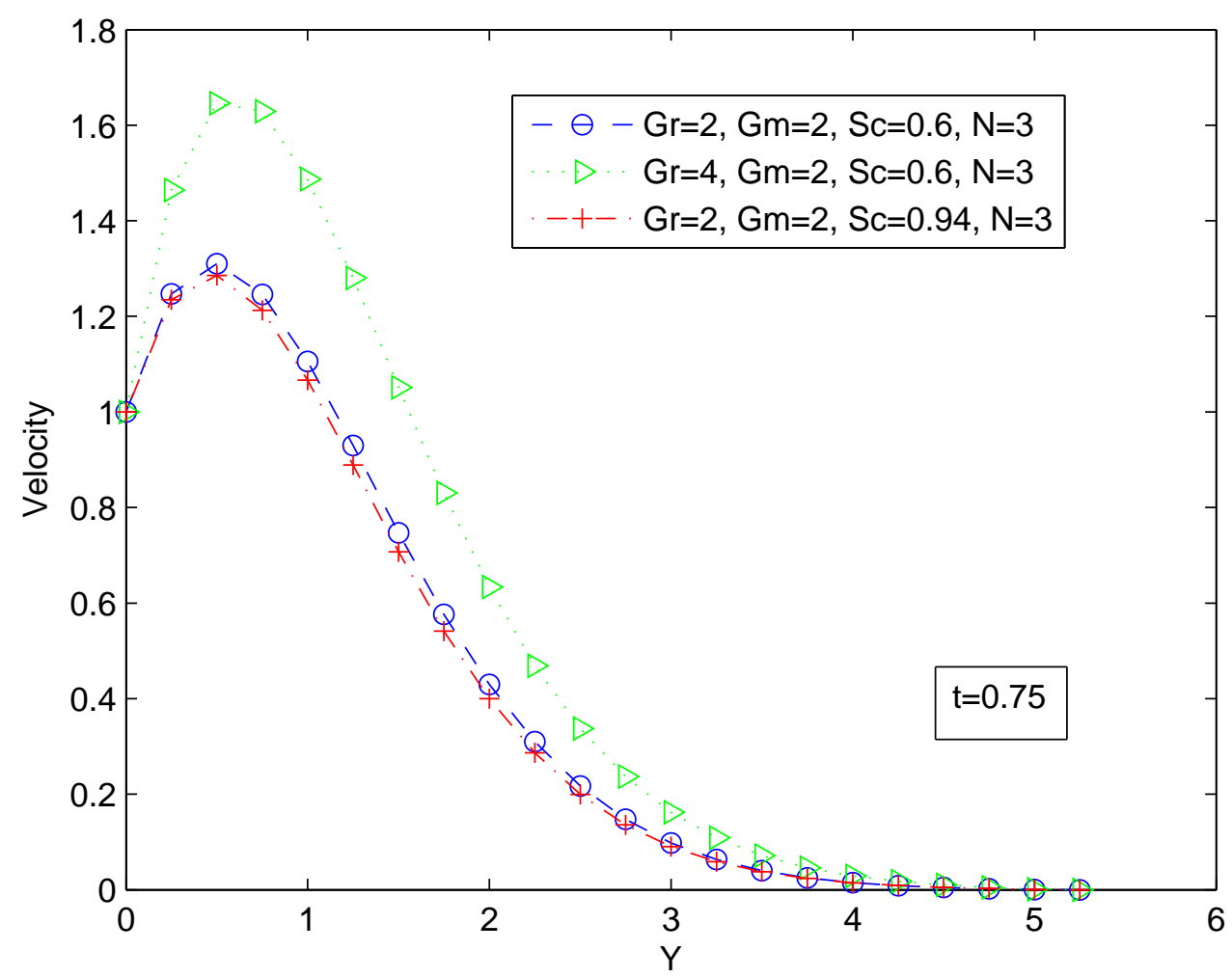

Figure 1. Velocity profile for various $\mathrm{Gr}, \mathrm{Gm}, \mathrm{Sc}$ and $\mathrm{N}$

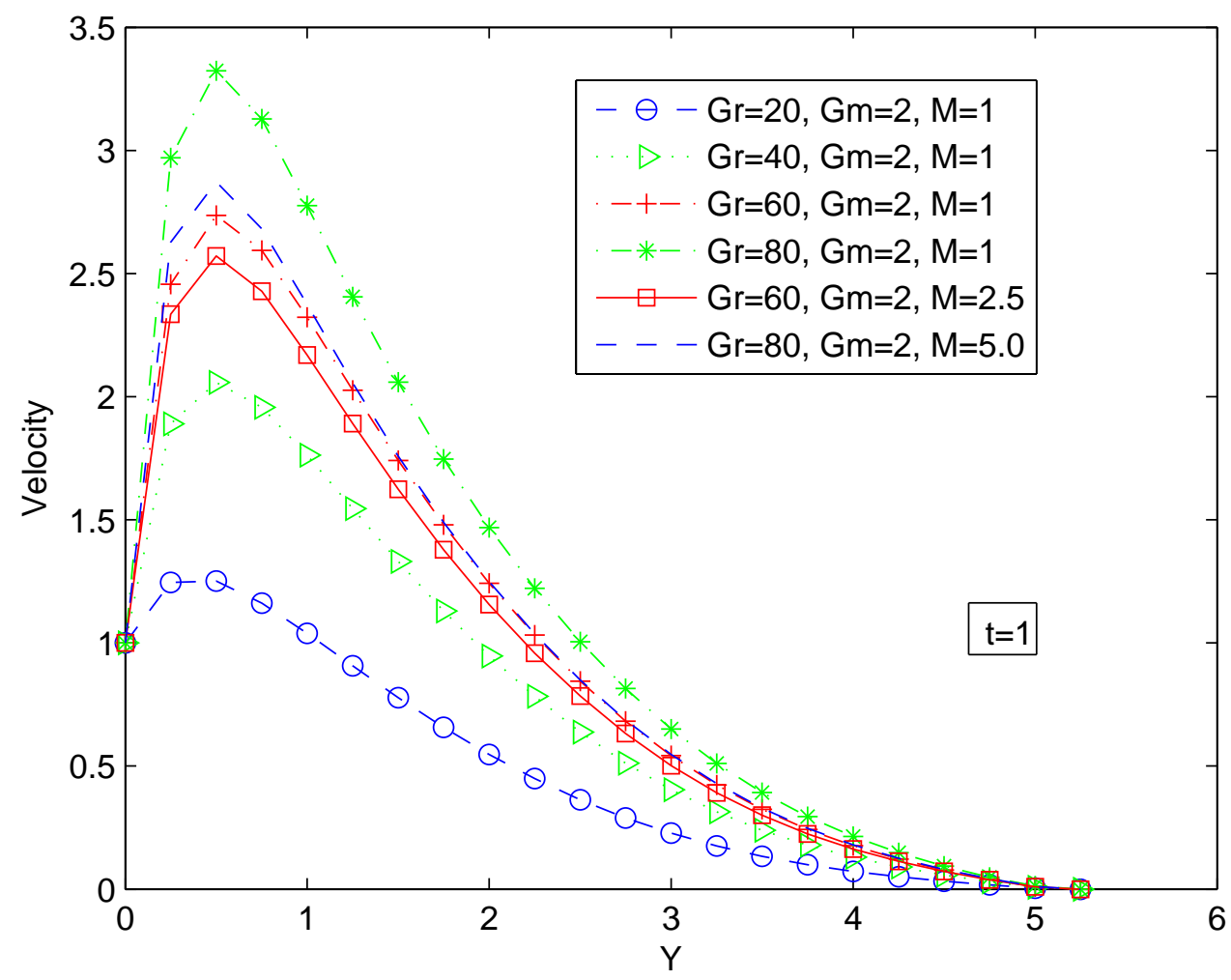

Figure 2. Effect of Gr, Gm and M on the Velocity profile 


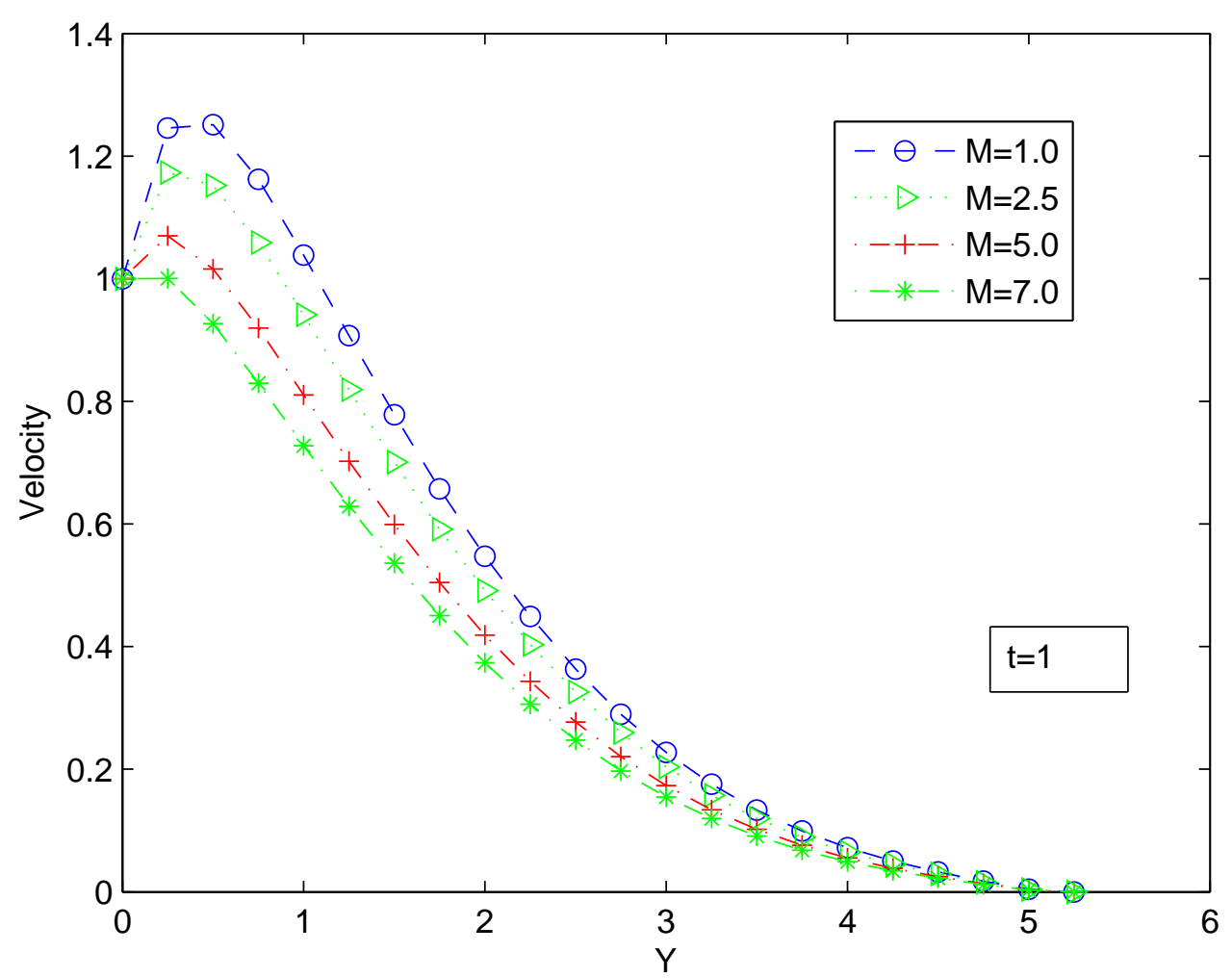

Figure 3. Effects of Magnetic field on velocity profile

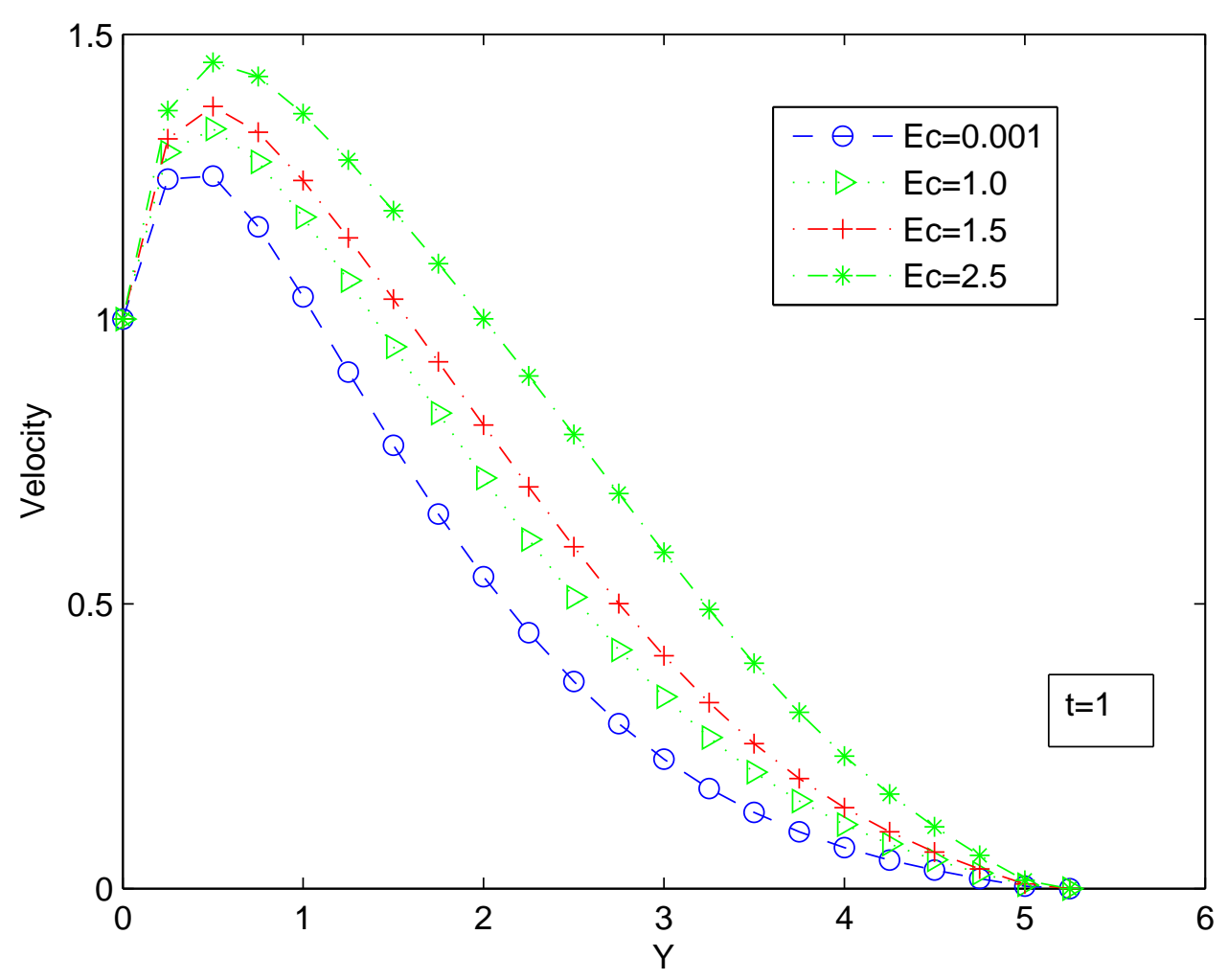

Figure 4. Velocity profile for values of Ec 


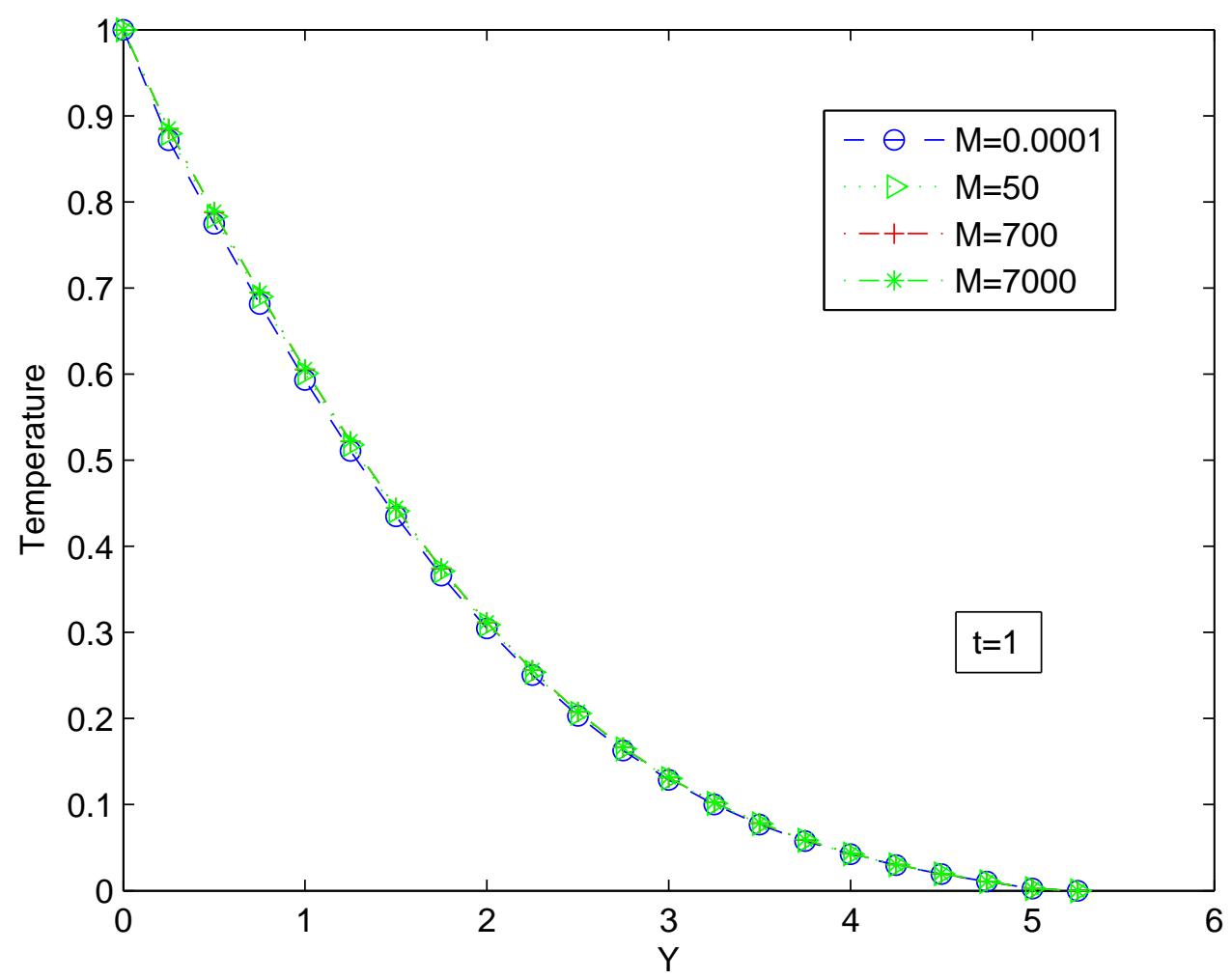

Figure 5. Effect of magnetic field on $\mathrm{T}$

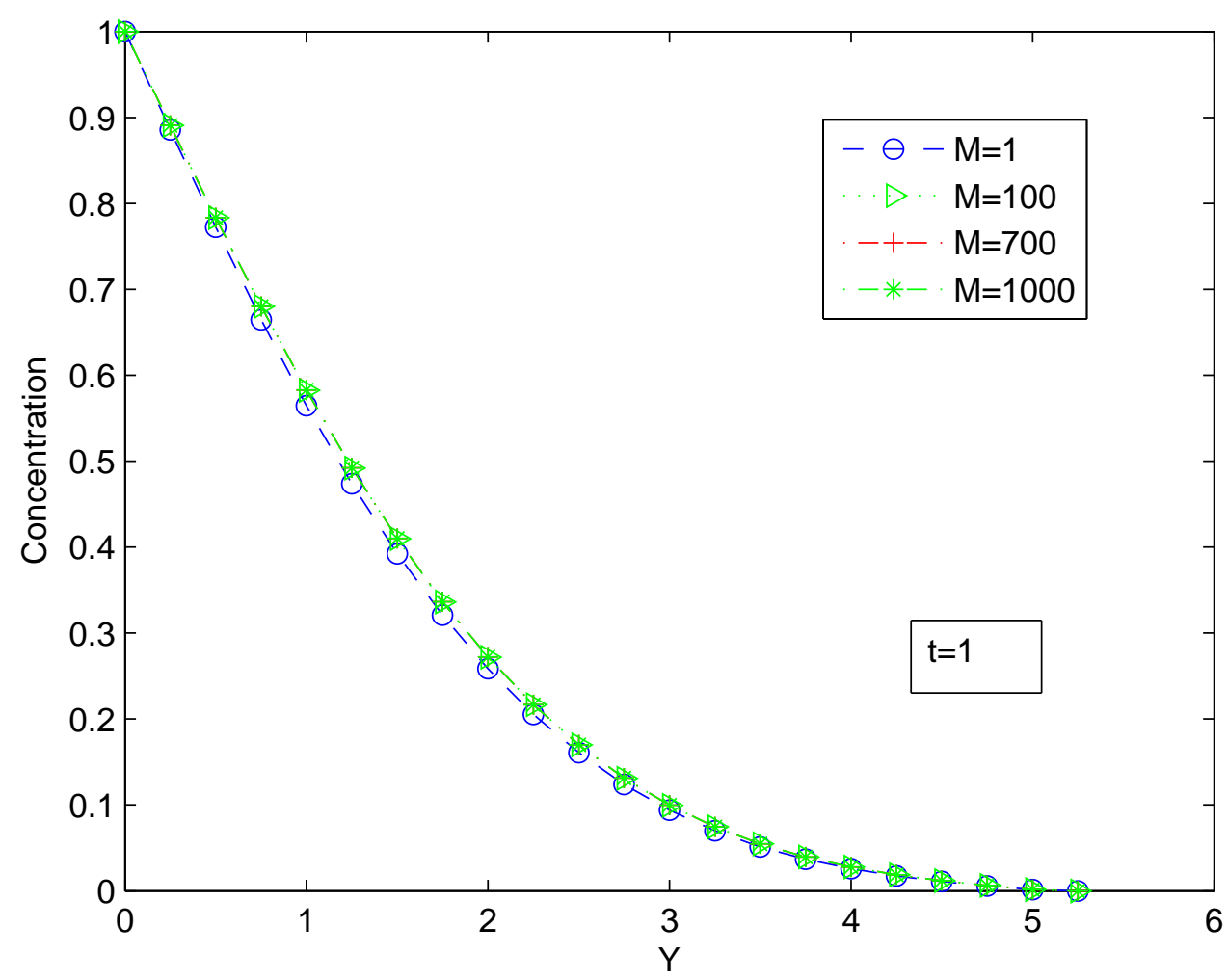

Figure 6. Effect of magnetic field on $\mathrm{C}$ 


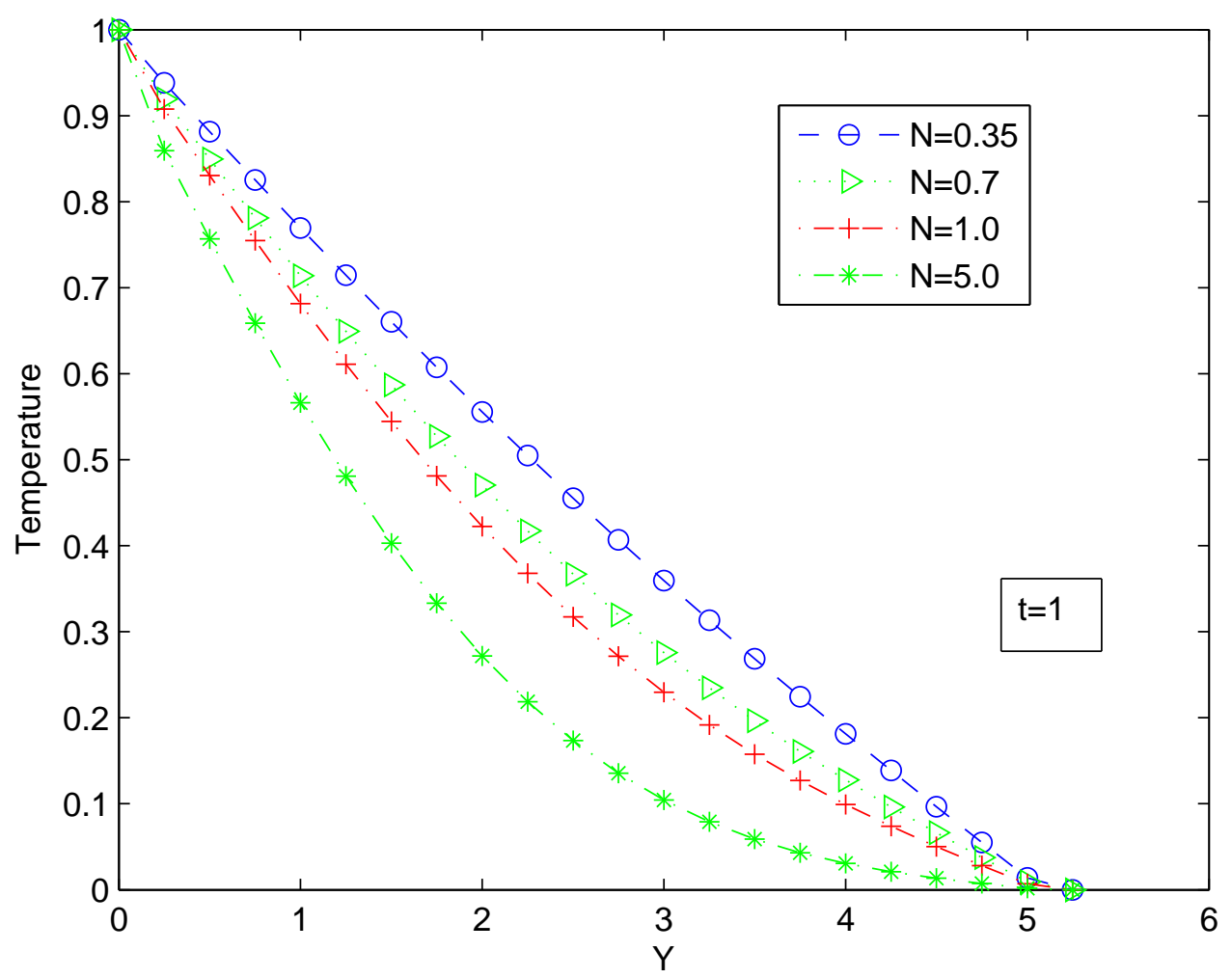

Figure 7. Temperature distribution with $\mathrm{Y}$ for various $\mathrm{N}$

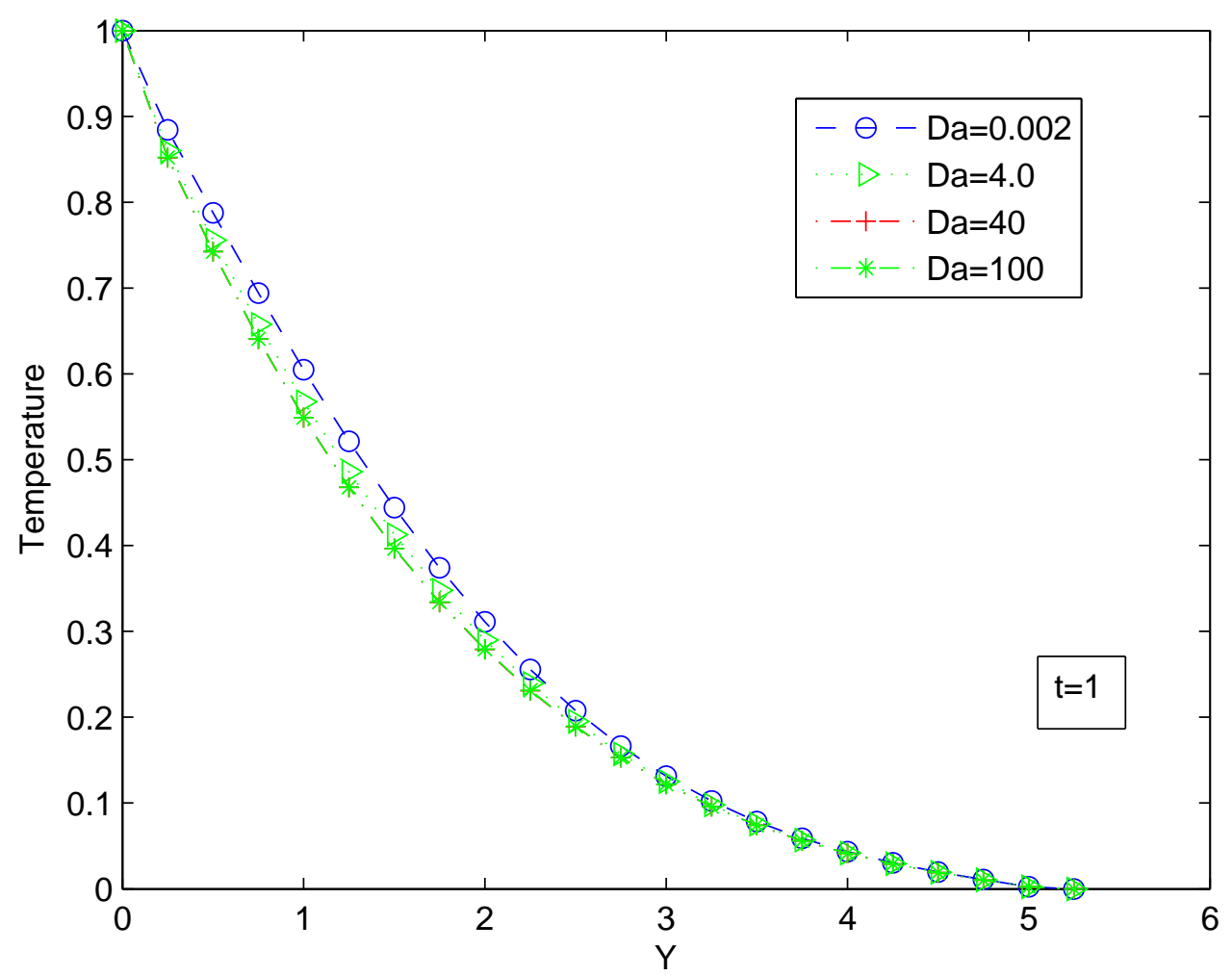

Figure 8. Temperature distribution for various Da 


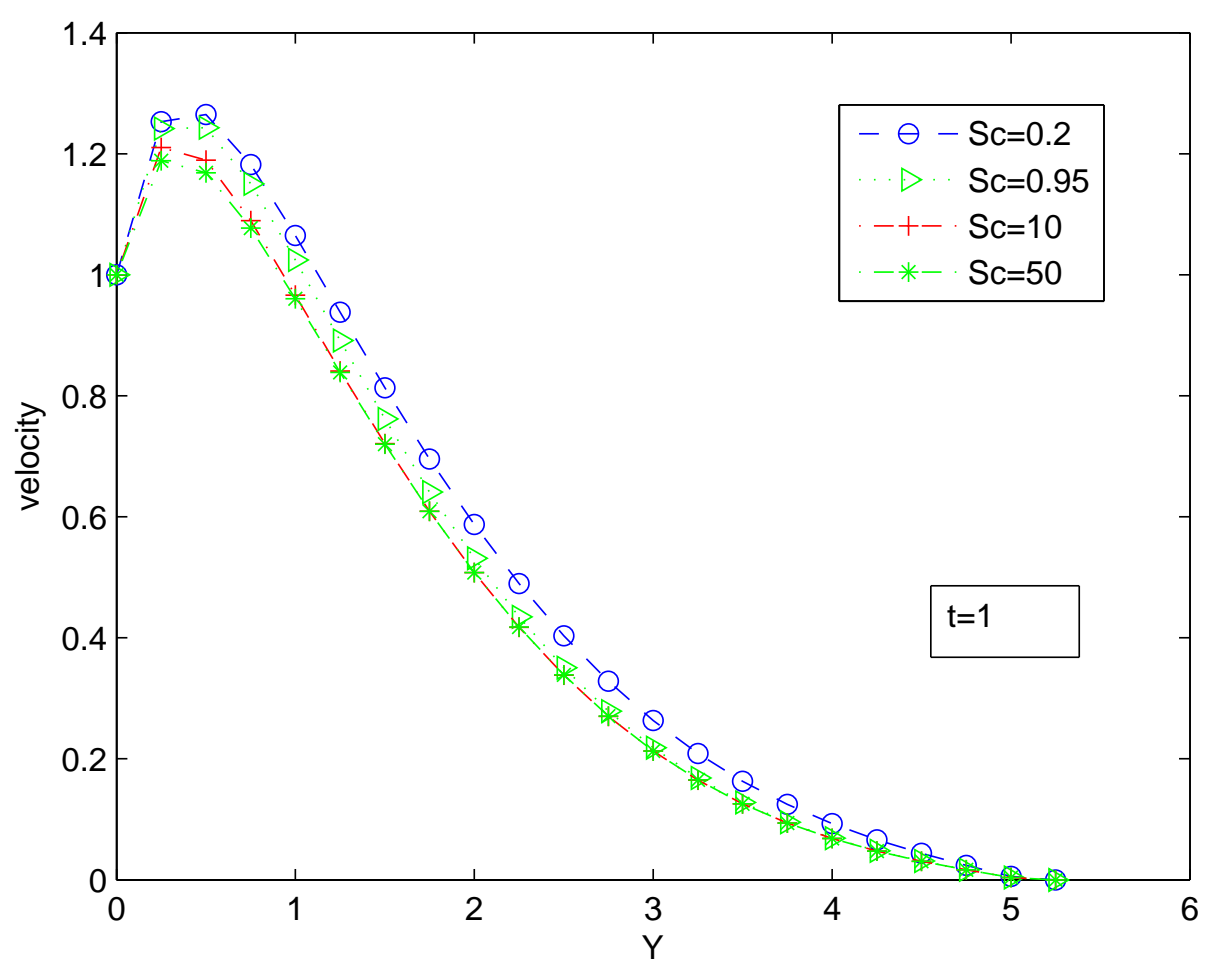

Figure 9. Velocity profile for various Sc values

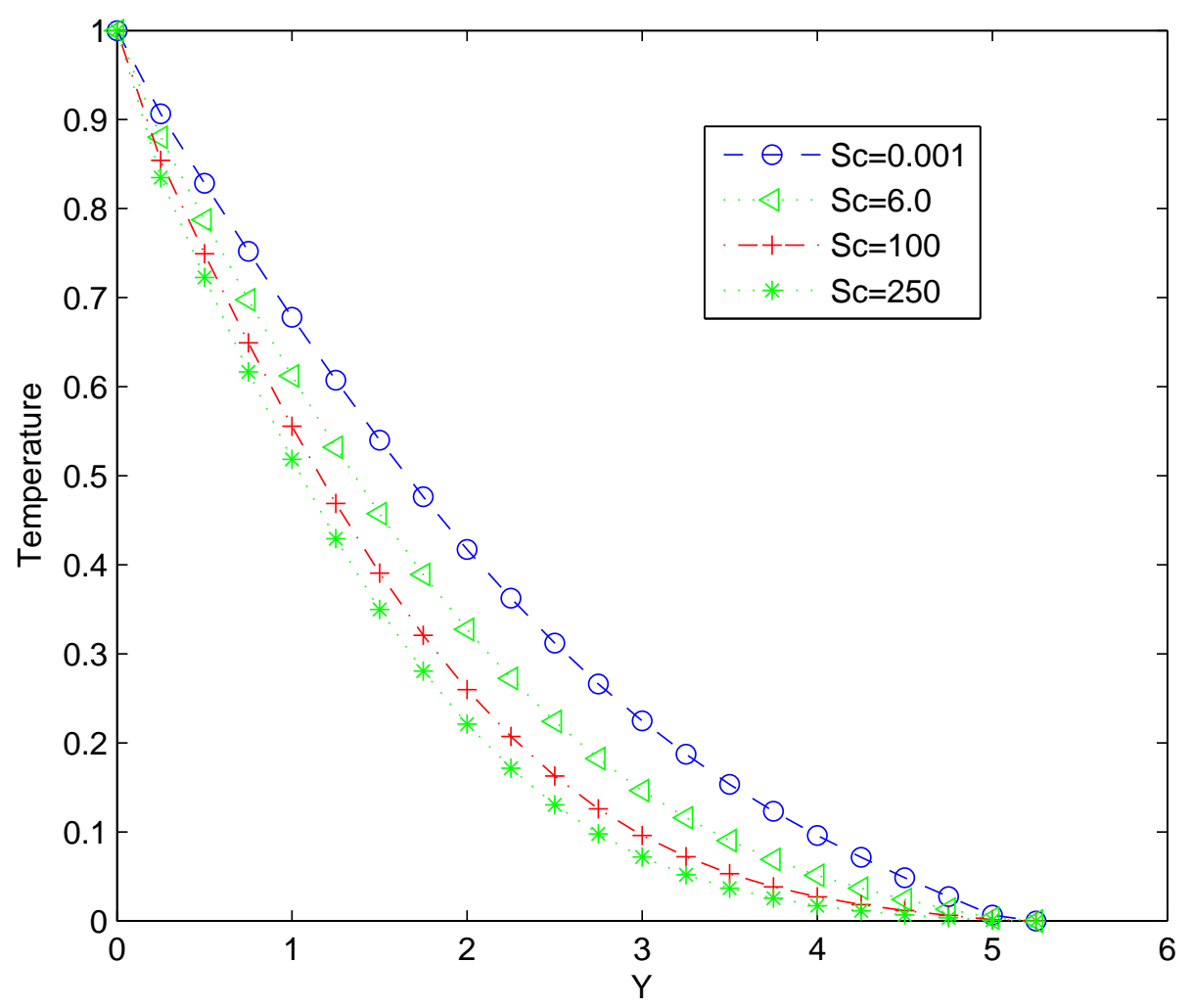

Figure 10. Temperature distribution with $\mathrm{X}$ for various Sc 


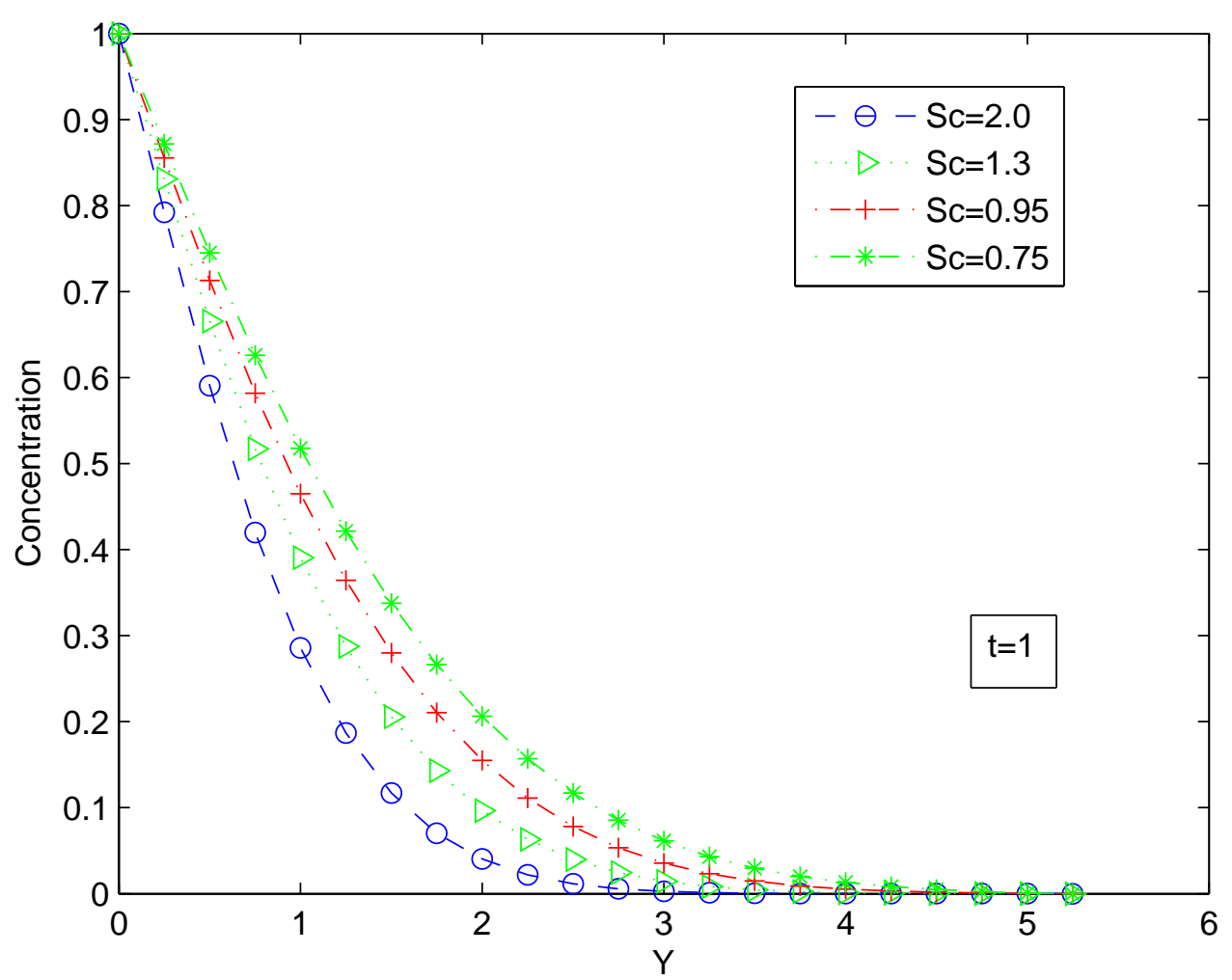

Figure 11. Concentration profile for various values of Sc

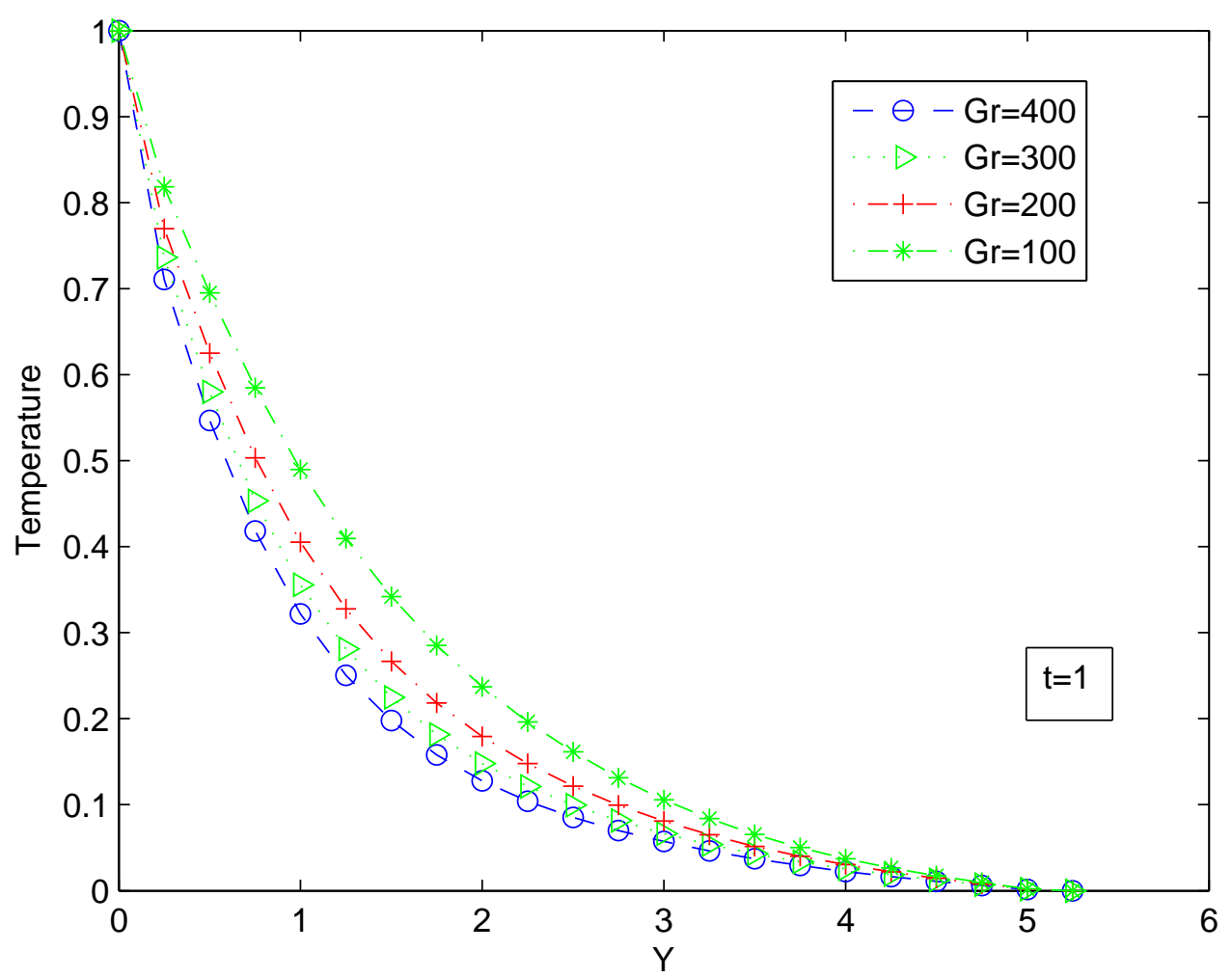

Figure 12. Temperature distribution for various values of $\mathrm{Gr}$ 


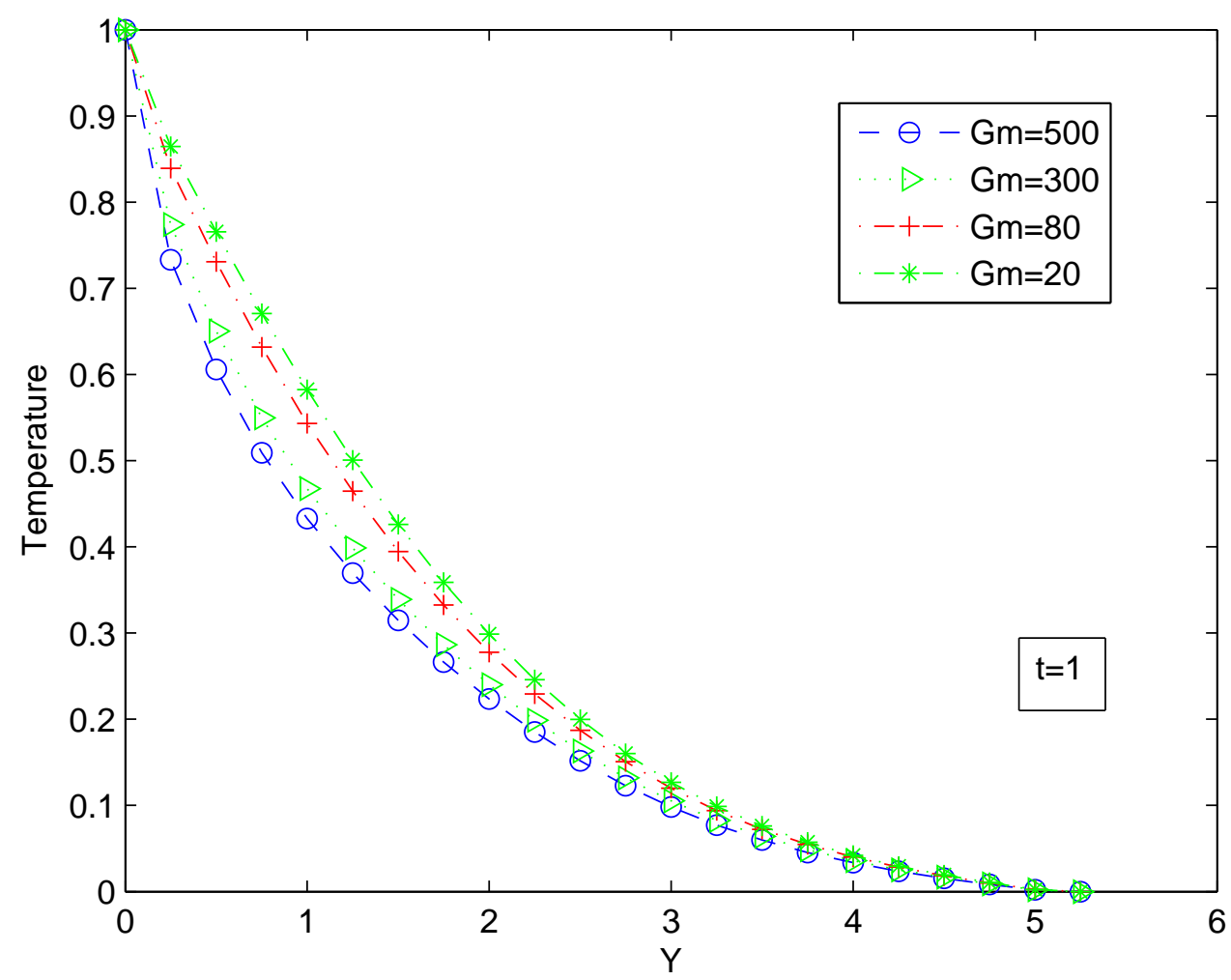

Figure 13. Temperature distribution for various values of $\mathrm{Gm}$

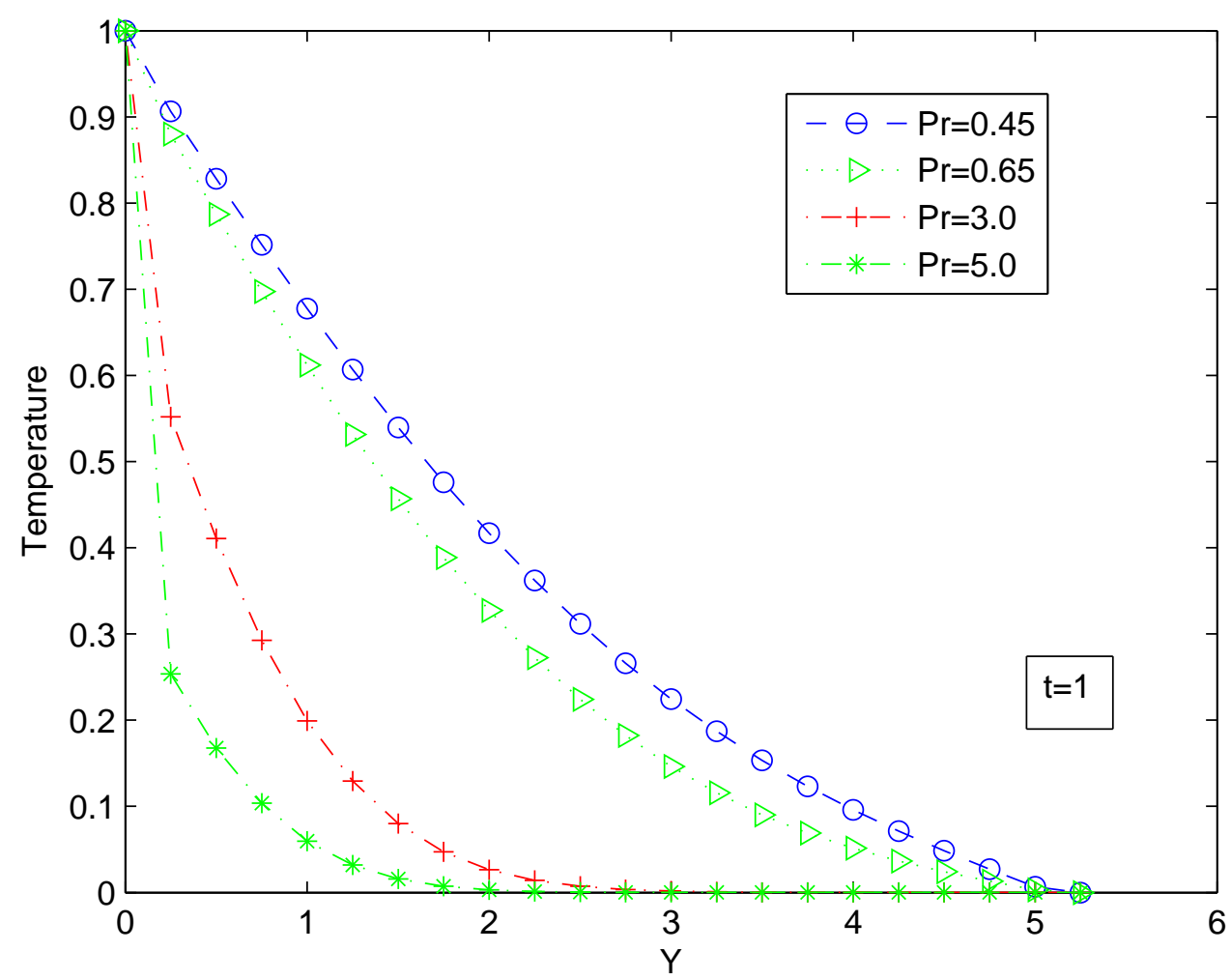

Figure 14. Temperature distribution for various values of $\operatorname{Pr}$ 


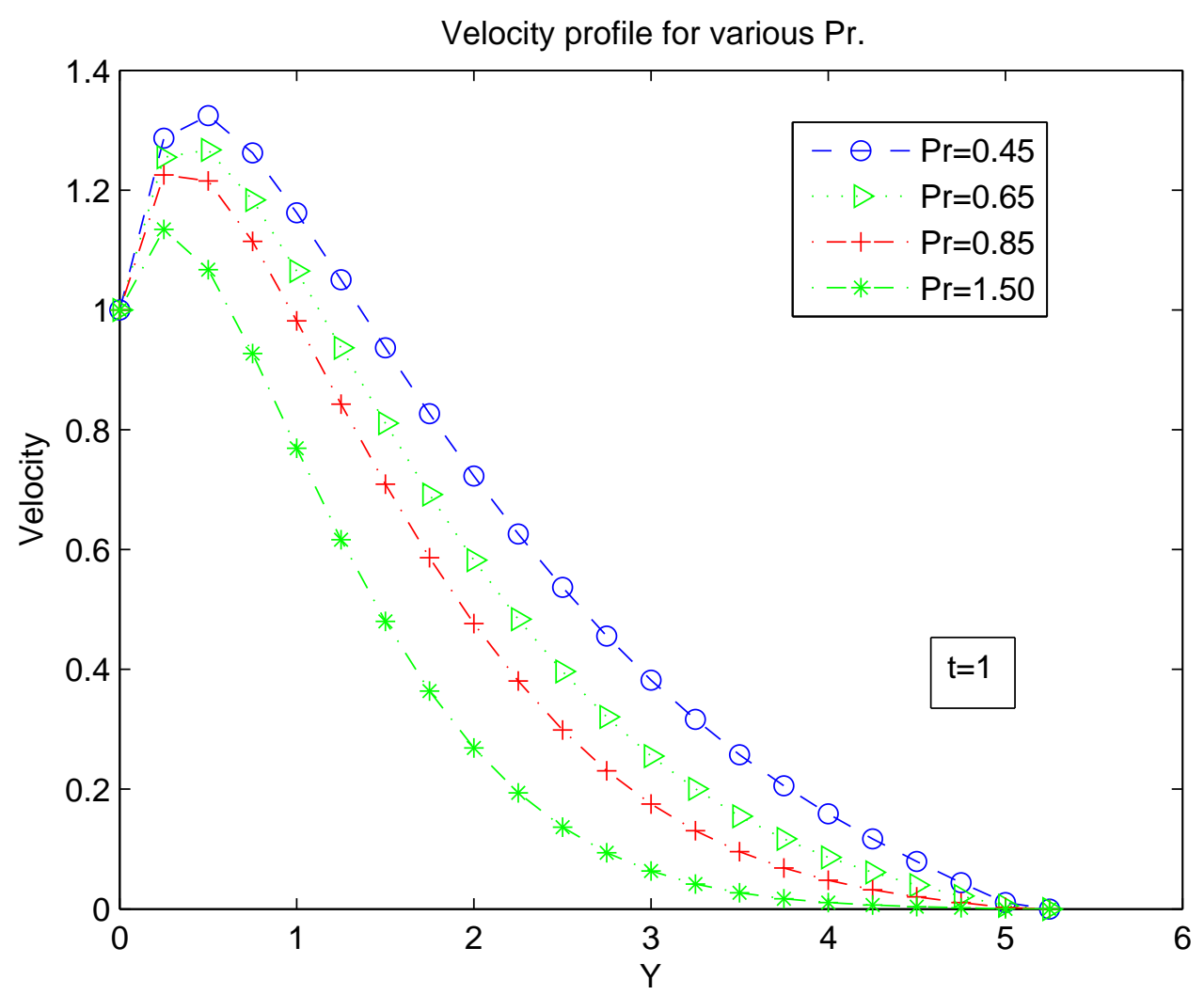

Figure 15. Velocity profile for values of Pr

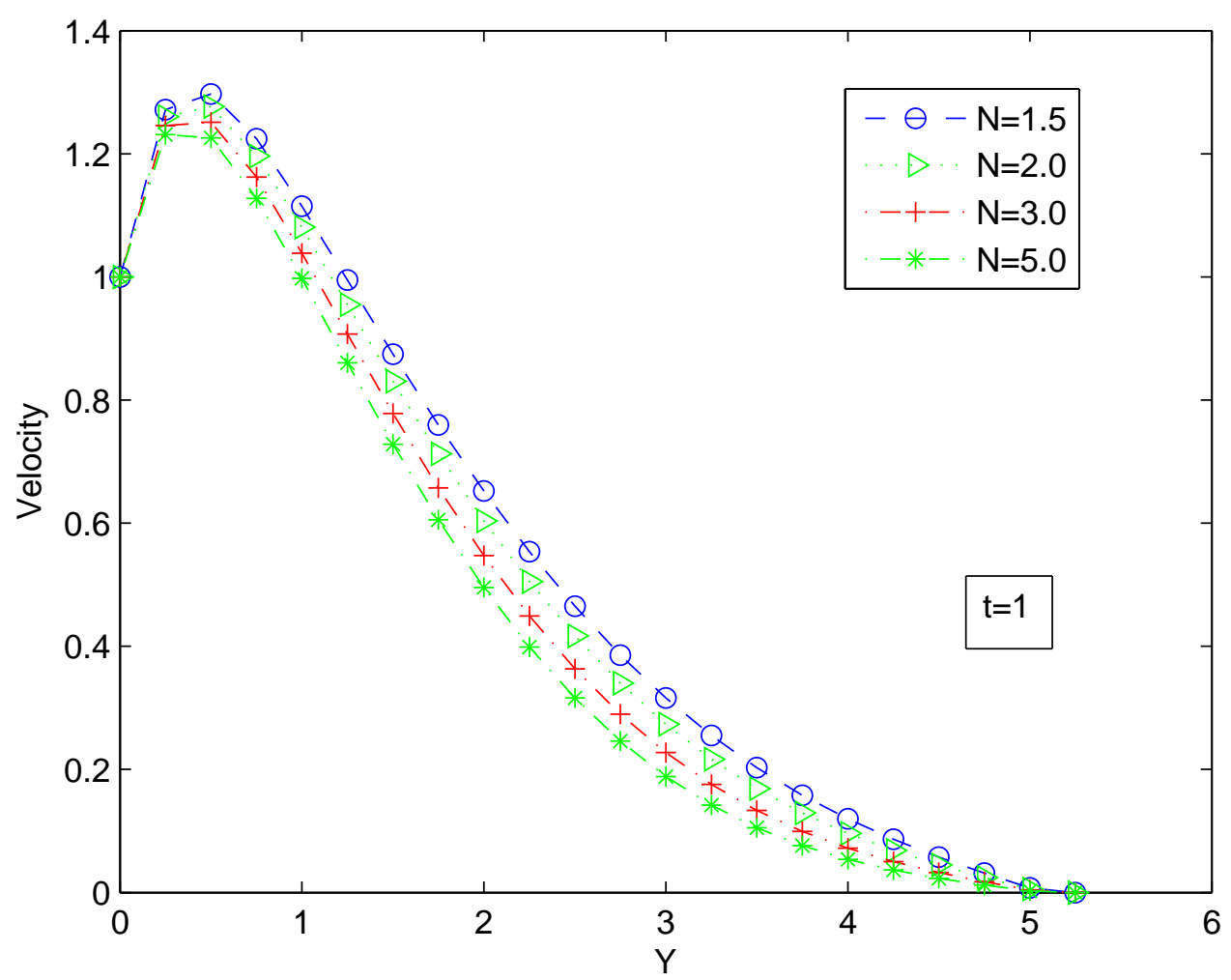

Figure 16.Velocity profile for values of $\mathrm{N}$ 


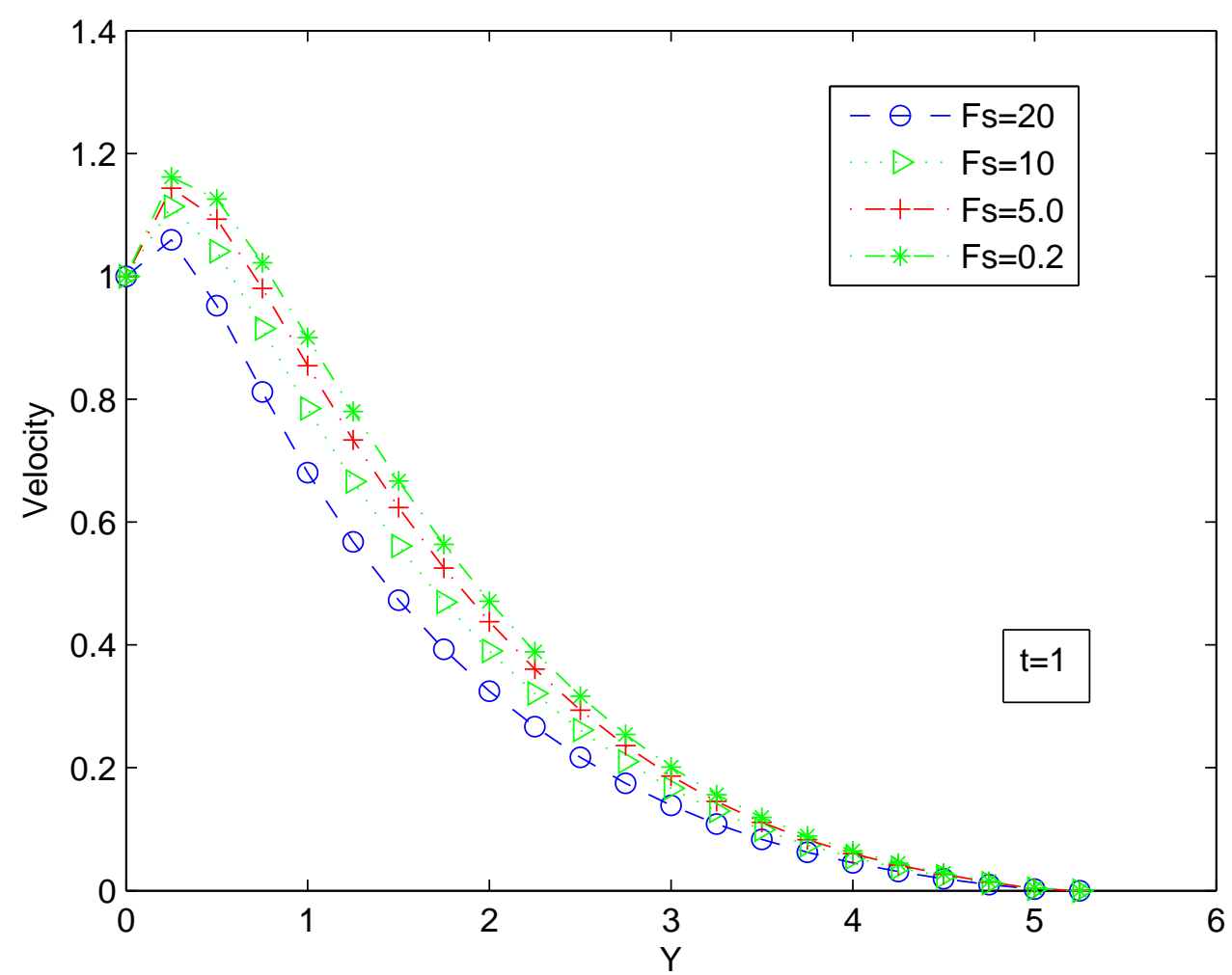

Figure 17. Velocity profile with $\mathrm{X}$ for various values of Fs

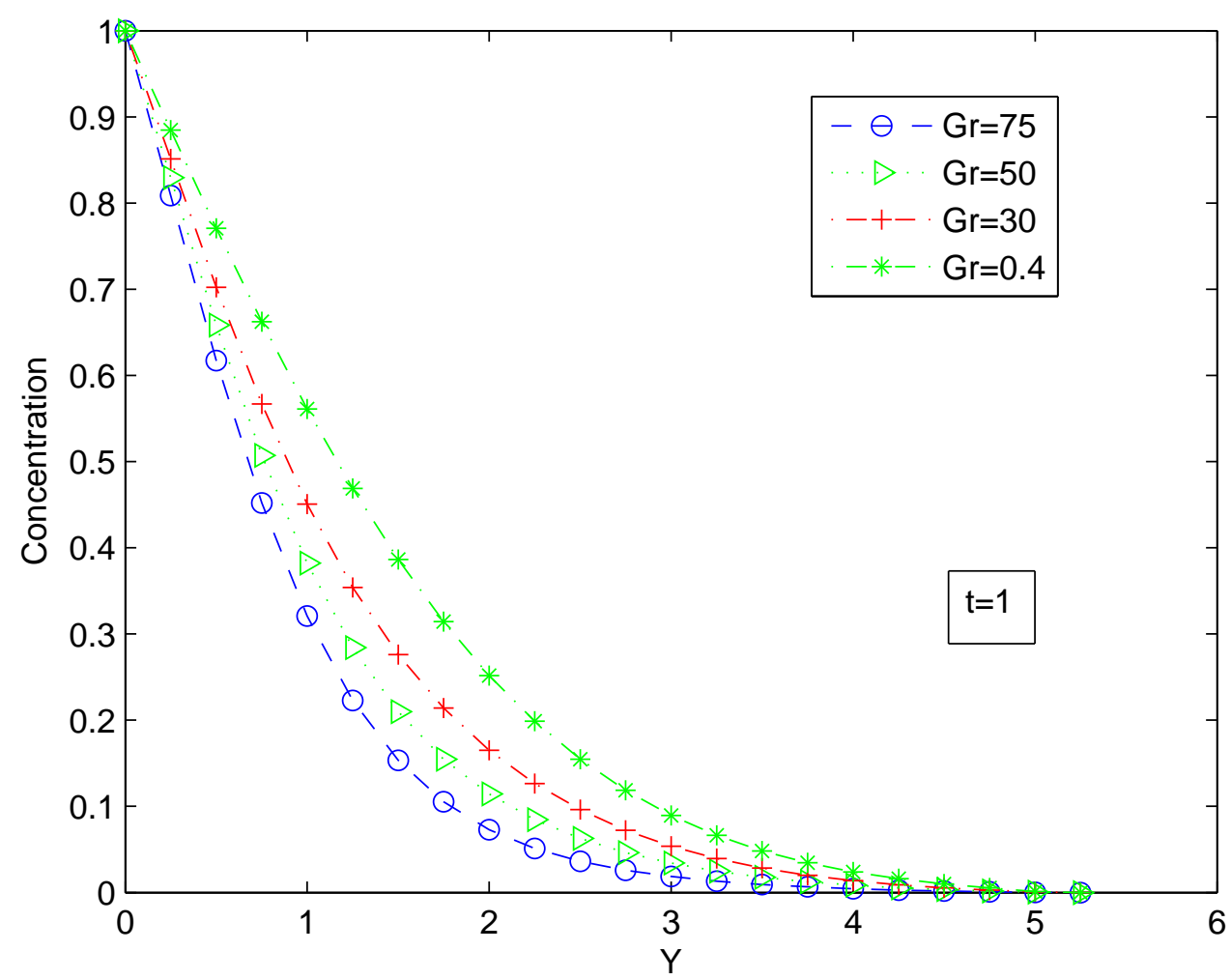

Figure 18. Concentration distribution for various values of $\mathrm{Gr}$ 


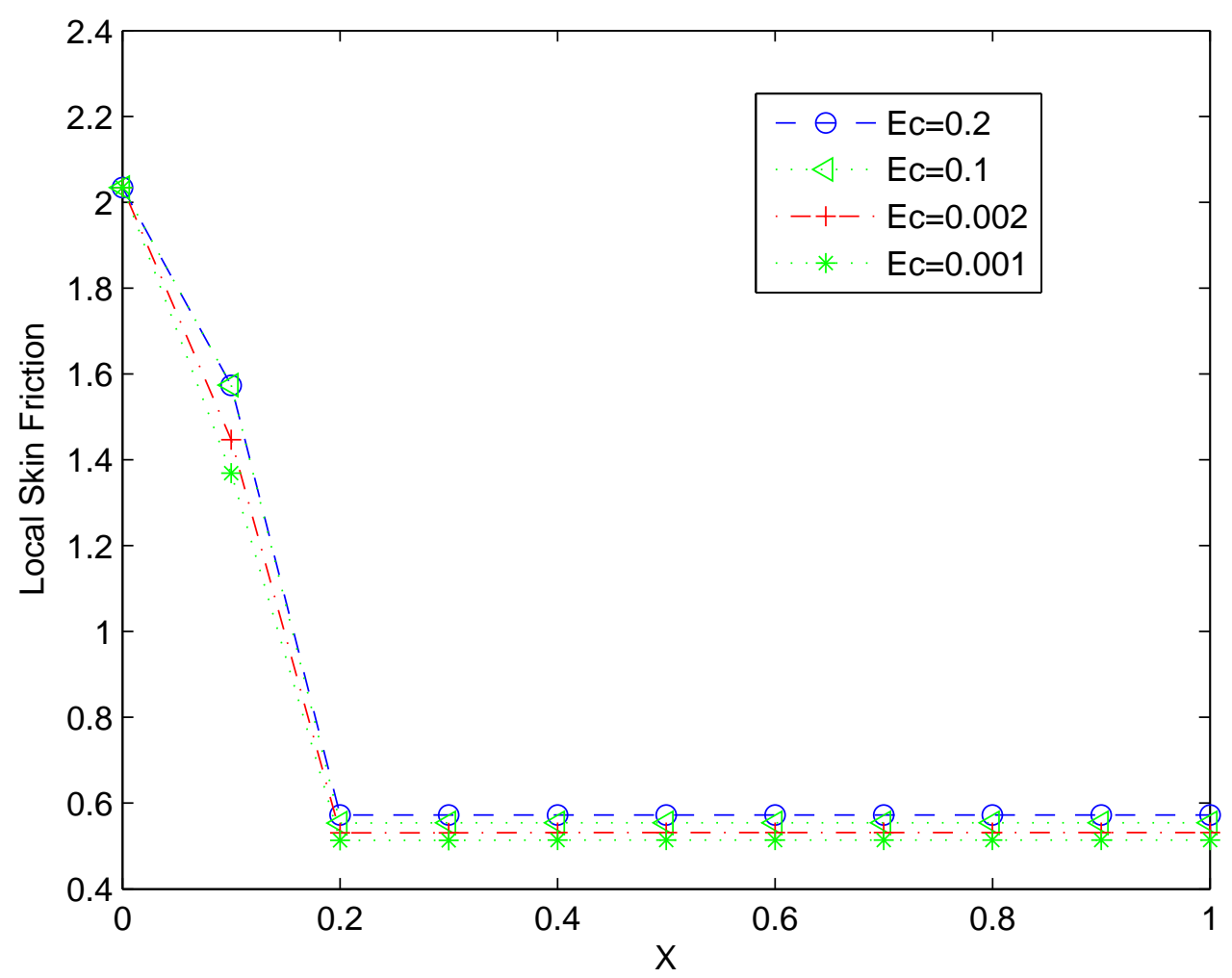

Figure 19. Local skin friction for various values of Ec

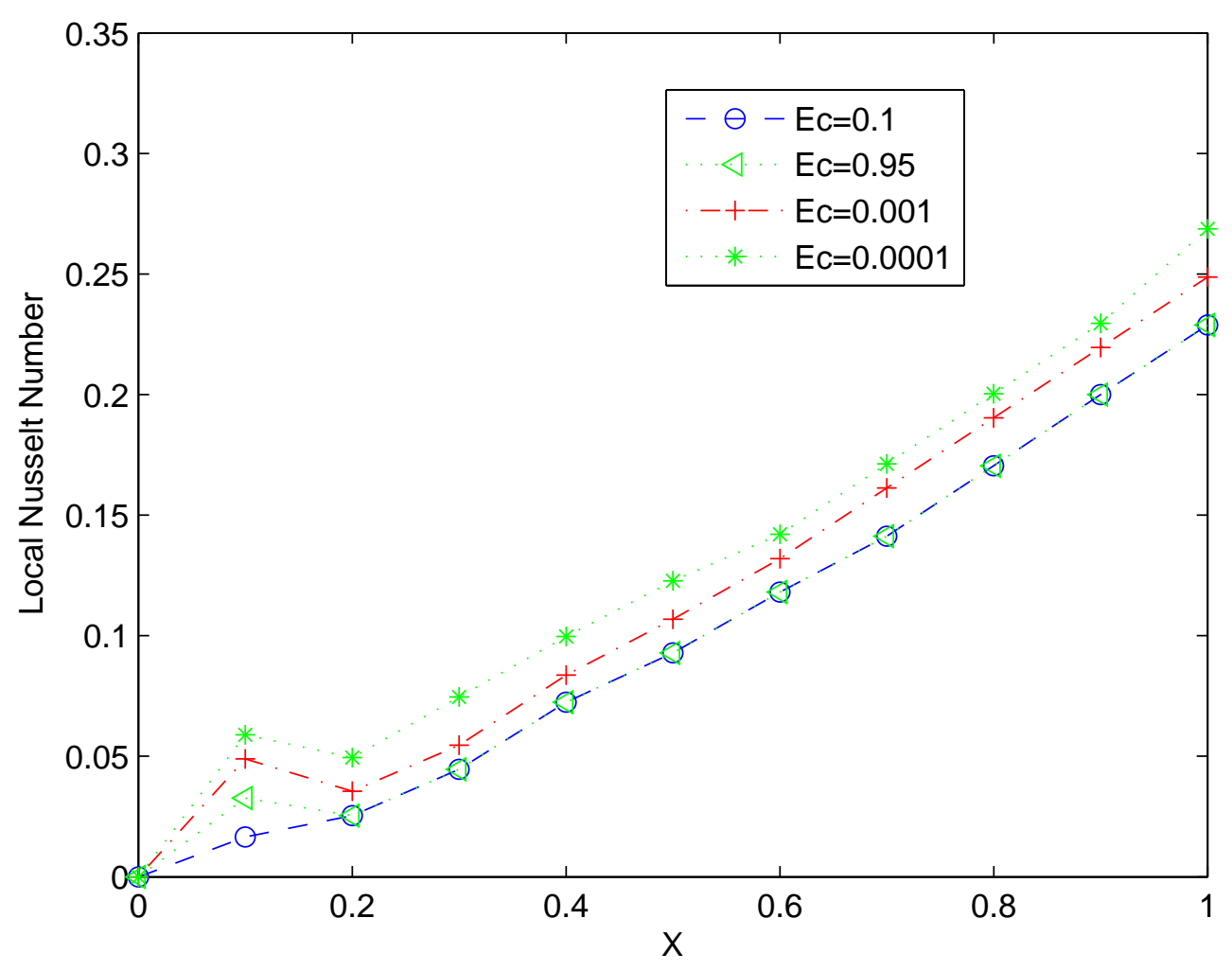

Figure 20. Local Nusselt number for various values of Ec 


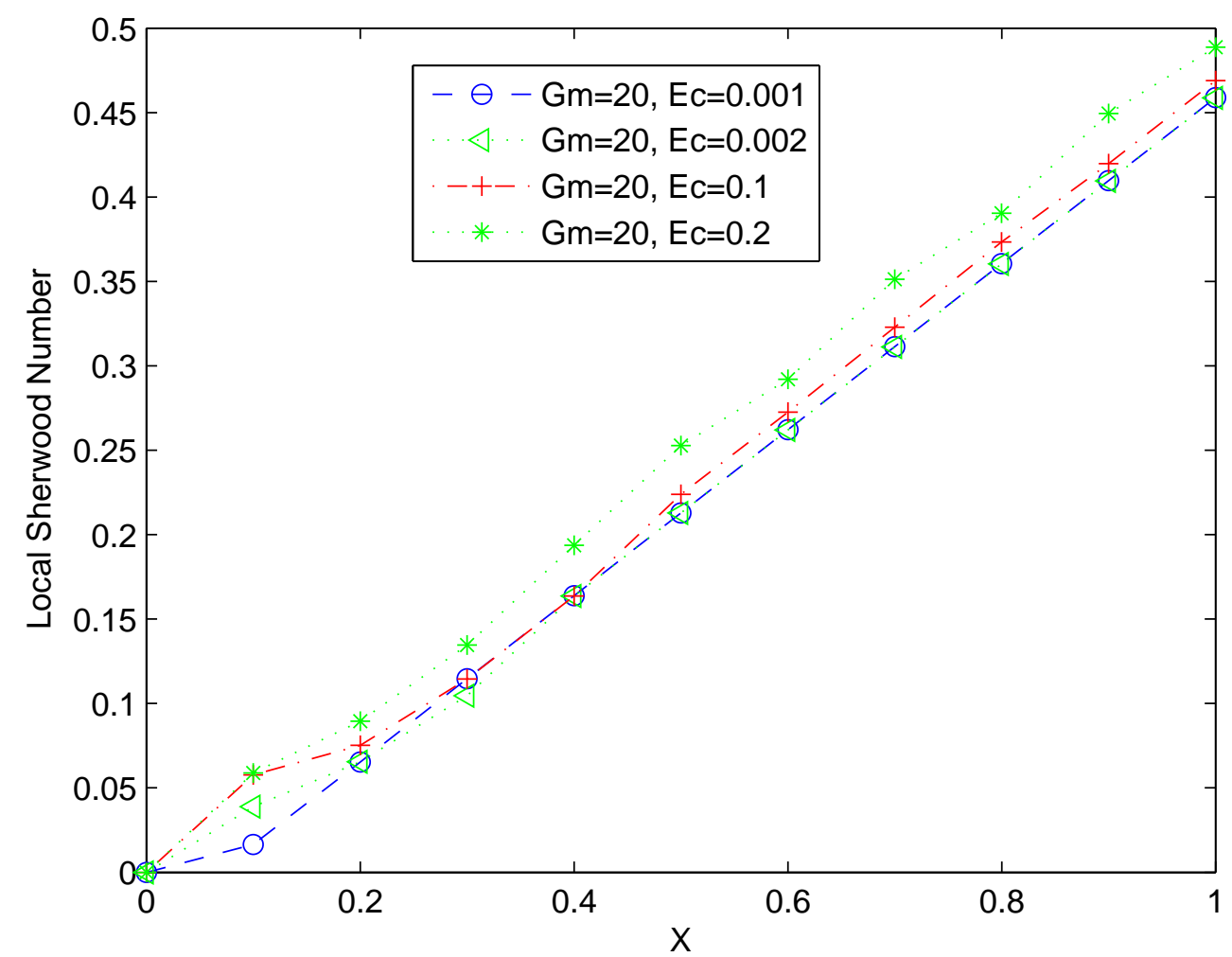

Figure 21. Local Sherwood number for various values of Gr, Gm and Ec 